\title{
The bryophyte flora of Akyazı, Arifiye, Geyve, Karapürçek districts (Sakarya, Turkey)
}

\author{
Güray UYAR *1, Muhammet ÖREN ${ }^{2}$ \& Mevlüt ALATAŞ ${ }^{3}$ \\ ORCID: 0000-0003-4038-6107; 0000-0003-1839-3087; 0000-0003-0862-0258
}

${ }^{1}$ Ankara Hacı Bayram Veli University, Polatlı Faculty of Science \& Arts, Depart. of Biology, 06900, Ankara, Turkey

${ }^{2}$ Zonguldak Bülent Ecevit University, Faculty of Science \& Arts, Department of Biology, 67100, Zonguldak, Turkey

${ }^{3}$ Munzur University, Faculty of Engineering, Department of Bioengineering, 62000, Tunceli, Turkey

\begin{abstract}
In this study, bryophyte flora of the Akyazı, Arifiye, Geyve, Karapürçek districts in Sakarya Province of Turkey was found out. A total of 1037 bryophyte specimens were collected from the research area in the different vegetation periods between 2016 and 2017. As a results of this study; 193 taxa (1 hornwort, 34 liverworts, 158 mosses) belonging to 113 genera and 55 families were determined. Among them, Sphagnum contortum Schultz is recorded for the second time from Turkey and also 11 taxa are new for the A2 grid-square according to the Turkish grid square system adopted by Henderson (1961). In addition, in the floristic list all taxa, with their Turkey distributions, which are determined by reviewing the related all literatures, and IUCN categories in European countries, moreover life forms of bryophytes and some ecological features such as; (humidity, light, acidity) are given.
\end{abstract}

Key words: bryophyte, flora, Akyazı, Arifiye, Geyve, Karapürçek

\section{Özet}

\section{Akyazı, Arifiye, Geyve, Karapürçek ilçeleri (Sakarya, Turkey) briyofit florası}

Bu çalışmada, Türkiye'nin Sakarya iline bağlı Akyazı, Arifiye, Geyve, Karapürçek ilçelerinin briyofit florası ortaya çıkartılmıştır. 2016 ve 2017 yılları arasında vejetasyonun farklı periyotlarında araştırma bölgesinden toplamda 1037 briyofit örneği toplandı. Bu çalışmanın sonucunda; 113 cins ve 55 familyaya ait olan 193 takson (1 boynuzotu, 34 ciğerotu, 158 karayosunu) belirlendi. Bunlar arasından, Sphagnum contortum Schultz Türkiye'den ikinci kez kayıt edildi ve ayrıca 11 takson da Henderson (1961) tarafından adapte edilen Türkiye kareleme sistemine göre A2 karesi için yenidir. Bununla birlikte, floristic listedeki tüm taksonlar onların ilgili bütün literatürlerin gözden geçirilmesi sonucu belirlenen, Türkiye dağılımları ve Avrupa ülkelerindeki IUCN kategorileri, ayrıca briyofitlerin yaşam formları ve bazı ekolojik özellikleri (nemlilik, ışık, asidite) ile birlikte verildi.

Anahtar kelimeler: briyofit, flora, Akyazı, Arifiye, Geyve, Karapürçek

\section{Introduction}

Plant scientists accept two kinds of land plants, respectively; bryophytes, and tracheophytes. Because of this, bryophytes are the second largest group of terrestrial plants and they are classified under Bryobiotina subkingdom in Plantae consist of mosses, hornworts and liverworts. At the same time, molecular phylogenetic studies concluded that bryophytes are oldest ancestors of existing land plants. [1]. So they give an idea about the migration of plants from aquatic environments to land. That's why a number of physical features link bryophytes to both land plants and aquatic plants. These different adaptations observed in Bryophytes also have allowed plants to colonize the terrestrial environments of the Earth. Because of this, they grow at various temperatures (cold poles and hot deserts), altitudes (from sea level to alpine) and humidity (from dry desserts to wet rainforests). At the same time, when habitat diversity increases, various

\footnotetext{
* Corresponding author / Haberleşmeden sorumlu yazar: Tel.: +905353067793; Fax.: +905353067793; E-mail: gurayuyar@ hotmail.com

(C) Copyright 2020 by Biological Diversity and Conservation

Received: 20.09.2020; Published: 15.04.2020

BioDiCon. 858-0919
} 
plant taxa, including bryophytes, are possible to emerge. This situation is seen more apparent in the geography of Turkey, due to Turkey's taking place at the junction of three phytogeographical regions (Euro-Siberian, Mediterranean and IranoTuranian) and their transition zones. Moreover, this habitat diversity causes both plentiful plant diversity and a high endemism rate of Turkey. Despite that there are many researches about Turkey's vascular flora, bryofloristic studies in Turkey have been unregarded for a long time. However, the important scientific gap under this topic have being filled with a series of accomplished field excursions and many new data. In addition, new bryophyte records were continuously added with each new study [2, 3, 4]. Apparently, bryophytes flora of Turkey still needs to work on more detailed researches. Therefore, we think that should be chosen as priority areas for bryophytes flora studies of natural forest areas in Turkey. That's why, in this study, Akyazi, Arifiye, Geyve, Karapürcek districts of Sakarya Province in Turkey were selected as the study area. These areas are situated to south and southeast of Sakarya Province represents a very special region for Turkey's ecology and has rich vegetation (Fig. 1). Unfortunately, there are only three published studies carried out in nearby the study area $[5,6,7]$. Because of this gap in the literature, this study will contribute to the study of the bryophytes flora and Turkey. And also we hope so it would be useful for future studies.

\subsection{Study area}

Akyazı, Arifiye, Geyve, Karapürçek districts of Sakarya province are located in Marmara region of Turkey and are surrounded with the districts Hendek in the northeast, Adapazar1 in, west, Sapanca in the northwest, Pamukova in the southwest, Taraklı in the south, Göynük, Mudurnu and Taşkesti districts of Bolu province in the southeast - east. Besides, the study area is located in A2 square, according to Henderson's grid system of Turkey [8], (Figure 1).

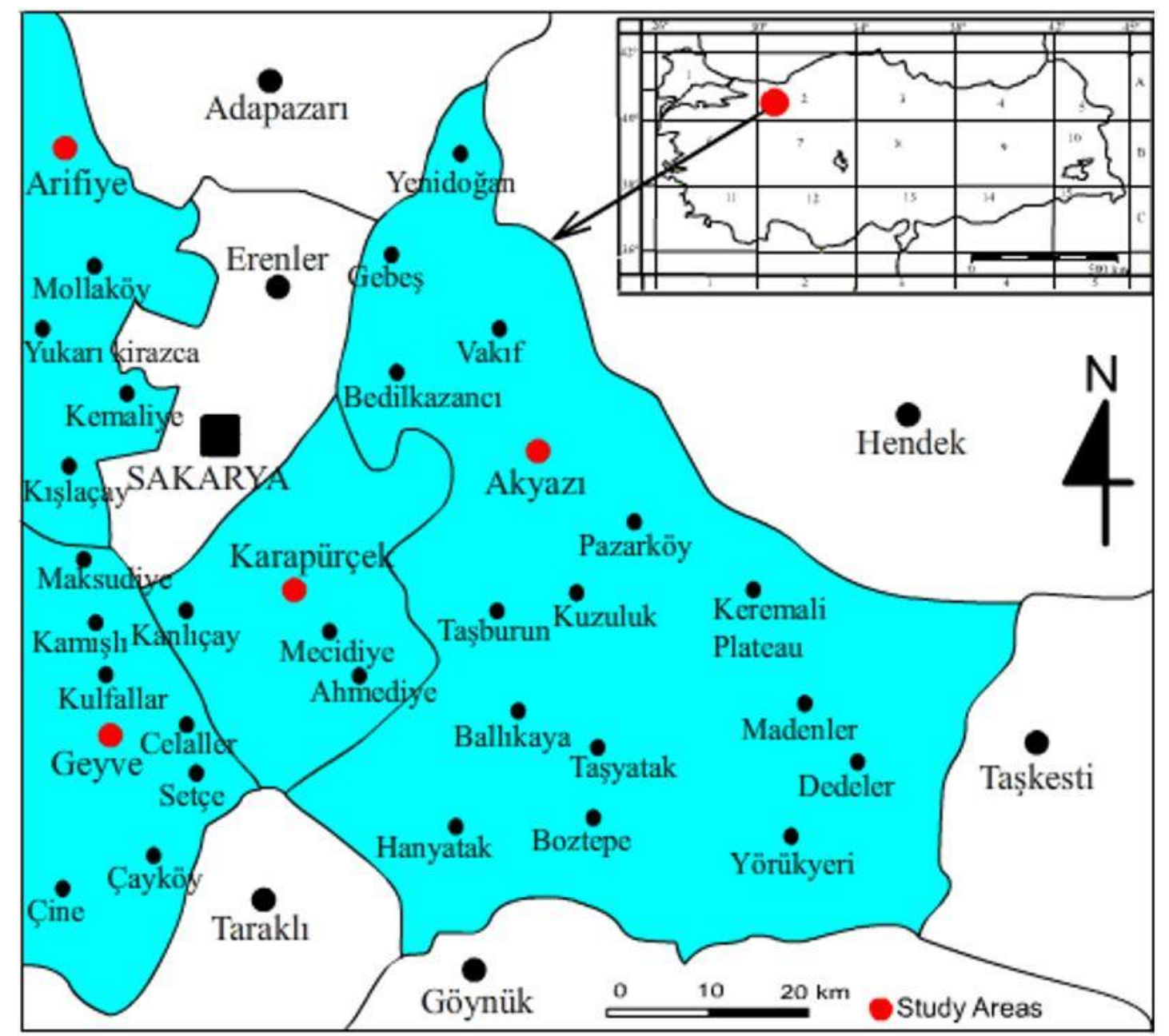

Figure 1. Grid system of Turkey adopted by Henderson (1961) and the study areas.

The climate of this area is similar to Marmara and the Black Sea climate. The air is humid in Sakarya and winters are usually rainy and mild while summers are hot. According to the amount of precipitation, the driest month is $41 \mathrm{~mm}$ in August and maximum rainfall month is $103 \mathrm{~mm}$ in December. In addition, the average annual precipitation is $754 \mathrm{~mm}$ and the yearly average temperature is $13.9{ }^{\circ} \mathrm{C}$ the lowest recorded temperature is $-14.5{ }^{0} \mathrm{C}$ and the highest recorded temperature is $41.8^{\circ} \mathrm{C}$. As a result of these climatic synthesis; the study area generally has a semi-arid and sometimes 
humid Mediterranean climate. [9, 10]. Moreover, this region is within the euxine province of the Euro-Siberian phytogeographic region [11]. In accordance with the region's climate the study area is primarily covered with pure and mixed deciduous forest (e.g. Fagus orientalis Lipsky, Carpinus betulus L., Tilia tomentosa Moench, Castanea sativa Mill., Alnus glutinosa (L.) Gaertn., Populus tremula L., Acer campestre L., Fraxinus ornus L., Platanus orientalis L. and Rhododendron ponticum L.,) in the northern slopes and stream beds and also conifers and maquis vegetation (e.g. Abies nordmanniana (Steven) Spach ssp. equi-trojani (Asch. \& Sint. ex Boiss.) Coode \& Cullen, Pinus sylvestris L., Pinus nigra Arn. subsp. pallasiana (Lamb) Hulmboe, Pinus brutia Ten., Quercus infectoria Oliver, Q. petraea (Matt.) Liebl., Laurus nobilis L., Erica arborea L., Corylus avellana L., Juniperus oxycedrus L.) in the western and southern slopes and also higher parts of the area [12].

\section{Material and methods}

The study was performed between the years 2016-2017. Bryophyte samples were collected from 54 locations, representing different habitats in the investigated region (Table 1), and also they were diagnosed by macroscopic morphology and microscopic characters using relevant literatures [13-25].

Table 1. Detailed informations about sampling points (LN: Locality number)

\begin{tabular}{|c|c|c|c|c|c|c|}
\hline L.N. & Districts & Locations & Altitude $m$ & \multicolumn{2}{|c|}{ GPS } & Date \\
\hline 1 & Karapürçek & Karapürçek & 542 & $40^{\circ} 36^{\prime} 25^{\prime \prime} \mathrm{N}$ & $30^{\circ} 27^{\prime} 41^{\prime \prime} \mathrm{E}$ & 28.10 .2016 \\
\hline 2 & Karapürçek & Karapürçek & 730 & $40^{\circ} 36^{\prime} 38^{\prime \prime} \mathrm{N}$ & $30^{\circ} 26^{\prime} 50^{\prime \prime} \mathrm{E}$ & 28.10 .2016 \\
\hline 3 & Karapürçek & Karapürçek & 862 & $40^{\circ} 36^{\prime} 26^{\prime \prime} \mathrm{N}$ & $30^{\circ} 26^{\prime} 11^{\prime \prime} \mathrm{E}$ & 28.10 .2016 \\
\hline 4 & Karapürçek & Upper parts of Kanlıçay & 321 & $40^{\circ} 37^{\prime} 56^{\prime \prime} \mathrm{N}$ & $30^{\circ} 29^{\prime} 39^{\prime \prime} \mathrm{E}$ & 28.10 .2016 \\
\hline 5 & Karapürçek & Karapürçek & 298 & $40^{\circ} 37^{\prime} 57^{\prime \prime} \mathrm{N}$ & $30^{\circ} 31^{\prime} 19^{\prime \prime} \mathrm{E}$ & 28.10 .2016 \\
\hline 6 & Karapürçek & Around of Mecidiye & 386 & $40^{\circ} 37^{\prime} 05^{\prime \prime} \mathrm{N}$ & $30^{\circ} 32^{\prime} 23^{\prime \prime} \mathrm{E}$ & 28.10 .2016 \\
\hline 7 & Akyazı & Bıçkıdere & 336 & $40^{\circ} 36^{\prime} 36^{\prime \prime} \mathrm{N}$ & $30^{\circ} 34^{\prime} 49^{\prime \prime} \mathrm{E}$ & 28.10 .2016 \\
\hline 8 & Akyazı & $\begin{array}{c}\text { The border of Akyazi-Yenidoğan, } \\
\text { Karagöl plateau }\end{array}$ & 1116 & $40^{\circ} 35^{\prime} 24^{\prime \prime} \mathrm{N}$ & $30^{\circ} 34^{\prime} 47^{\prime \prime} \mathrm{E}$ & 29.10 .2016 \\
\hline 9 & Akyazı & Between Karagöl plateau and Karagöl & 1236 & $40^{\circ} 30^{\prime} 54^{\prime \prime} \mathrm{N}$ & $30^{\circ} 33^{\prime} 56^{\prime \prime} \mathrm{E}$ & 29.10 .2016 \\
\hline 10 & Akyazı & Acelle plateau & 1140 & $40^{\circ} 30^{\prime} 54^{\prime \prime} \mathrm{N}$ & $30^{\circ} 37^{\prime} 02^{\prime \prime} \mathrm{E}$ & 29.10 .2016 \\
\hline 11 & Akyazı & Around of Hasanbey & 475 & $40^{\circ} 39^{\prime} 51^{\prime \prime} \mathrm{N}$ & $30^{\circ} 40^{\prime} 04^{\prime \prime} \mathrm{E}$ & 20.05.2017 \\
\hline 12 & Akyazı & Around of Güzlek village & 765 & $40^{\circ} 39^{\prime} 11^{\prime \prime} \mathrm{N}$ & $30^{\circ} 42^{\prime} 30^{\prime \prime} \mathrm{E}$ & 20.05.2017 \\
\hline 13 & Akyazı & Keremali plateau & 1200 & $40^{\circ} 38^{\prime} 44^{\prime \prime} \mathrm{N}$ & $30^{\circ} 45^{\prime} 15^{\prime \prime} \mathrm{E}$ & 20.05.2017 \\
\hline 14 & Akyazı & Mausoleum of Keremali & 1520 & $40^{\circ} 39^{\prime} 04^{\prime \prime} \mathrm{N}$ & $30^{\circ} 45^{\prime} 18^{\prime \prime} \mathrm{E}$ & 20.05.2017 \\
\hline 15 & Akyazı & Around of Çamlıca Lake & 1168 & $40^{\circ} 40^{\prime} 06^{\prime \prime} \mathrm{N}$ & $30^{\circ} 46^{\prime} 16^{\prime \prime} \mathrm{E}$ & 20.05.2017 \\
\hline 16 & Akyazı & Between Çamlıca and Gölyayla & 1080 & $40^{\circ} 40^{\prime} 32^{\prime \prime} \mathrm{N}$ & $30^{\circ} 45^{\prime} 51^{\prime \prime} \mathrm{E}$ & 20.05.2017 \\
\hline 17 & Akyazı & Between Dedeler and Madenler & 477 & $40^{\circ} 35^{\prime} 21^{\prime \prime} \mathrm{N}$ & $30^{\circ} 49^{\prime} 28^{\prime \prime} \mathrm{E}$ & 21.05.2017 \\
\hline 18 & Akyazı & Turnalı plateau route & 1152 & $40^{\circ} 36^{\prime} 46^{\prime \prime} \mathrm{N}$ & $30^{\circ} 48^{\prime} 30^{\prime \prime} \mathrm{E}$ & 21.05.2017 \\
\hline 19 & Akyazı & Turnalı plateau & 1375 & $40^{\circ} 37^{\prime} 44^{\prime \prime} \mathrm{N}$ & $30^{\circ} 49^{\prime} 28^{\prime \prime} \mathrm{E}$ & 21.05.2017 \\
\hline 20 & Akyazı & Turnalı plateau & 1410 & $40^{\circ} 37^{\prime} 54^{\prime \prime} \mathrm{N}$ & $30^{\circ} 49^{\prime} 51^{\prime \prime} \mathrm{E}$ & 21.05.2017 \\
\hline 21 & Akyaz1 & Around of Yeniyayla & 1471 & $40^{\circ} 38^{\prime} 40^{\prime \prime} \mathrm{N}$ & $30^{\circ} 50^{\prime} 57^{\prime \prime} \mathrm{E}$ & 21.05.2017 \\
\hline 22 & Akyazı & Yeniyayla plateau & 1535 & $40^{\circ} 39^{\prime} 13^{\prime \prime} \mathrm{N}$ & $30^{\circ} 51^{\prime} 25^{\prime \prime} \mathrm{E}$ & 21.05.2017 \\
\hline 23 & Akyazı & Sultanpınarı plateau & 1135 & $40^{\circ} 32^{\prime} 16^{\prime \prime} \mathrm{N}$ & $30^{\circ} 40^{\prime} 56^{\prime \prime} \mathrm{E}$ & 22.05.2017 \\
\hline 24 & Akyazı & $\begin{array}{c}\text { Between Sultanpinarı and } \\
\text { Acelle plateau }\end{array}$ & 1283 & $40^{\circ} 30^{\prime} 49^{\prime \prime} \mathrm{N}$ & $30^{\circ} 39^{\prime} 47^{\prime \prime} \mathrm{E}$ & 22.05 .2017 \\
\hline 25 & Akyazı & Acelle plateau, near Lake & 1215 & $40^{\circ} 30^{\prime} 22^{\prime \prime} \mathrm{N}$ & $30^{\circ} 40^{\prime} 15^{\prime \prime} \mathrm{E}$ & 22.05.2017 \\
\hline 26 & Akyazı & $\begin{array}{c}\text { Between Acelle plateau and } \\
\text { Hanyatak }\end{array}$ & 1306 & $40^{\circ} 29^{\prime} 26^{\prime \prime} \mathrm{N}$ & $30^{\circ} 41^{\prime} 10^{\prime \prime} \mathrm{E}$ & 22.05 .2017 \\
\hline 27 & Akyazı & The way of Boztepe & 1355 & $40^{\circ} 29^{\prime} 25^{\prime \prime} \mathrm{N}$ & $30^{\circ} 44^{\prime} 18^{\prime \prime} \mathrm{E}$ & 22.05.2017 \\
\hline
\end{tabular}


Table 1. Continued

\begin{tabular}{|c|c|c|c|c|c|c|}
\hline 28 & Akyazı & Around of Yörükyeri & 1285 & $40^{\circ} 31^{\prime} 05^{\prime \prime} \mathrm{N}$ & $30^{\circ} 46^{\prime} 16^{\prime \prime} \mathrm{E}$ & 22.05.2017 \\
\hline 29 & Akyazı & Between Yörükyeri and Haydarlar & 761 & $40^{\circ} 33^{\prime} 15^{\prime \prime} \mathrm{N}$ & $30^{\circ} 46^{\prime} 51^{\prime \prime} \mathrm{E}$ & 22.05 .2017 \\
\hline 30 & Akyazı & $\begin{array}{l}\text { Between Pazarköy and } \\
\text { Merkezyeniköy }\end{array}$ & 232 & $40^{\circ} 39^{\prime} 20^{\prime \prime} \mathrm{N}$ & $30^{\circ} 39^{\prime} 42^{\prime \prime} \mathrm{E}$ & 24.05.2017 \\
\hline 31 & Akyazı & Around of Merkezyeniköy & 371 & $40^{\circ} 38^{\prime} 58^{\prime \prime} \mathrm{N}$ & $30^{\circ} 38^{\prime} 55^{\prime \prime} \mathrm{E}$ & 24.05.2017 \\
\hline 32 & Akyazı & Around of Kuzuluk & 195 & $40^{\circ} 38^{\prime} 23^{\prime \prime} \mathrm{N}$ & $30^{\circ} 38^{\prime} 52^{\prime \prime} \mathrm{E}$ & 24.05.2017 \\
\hline 33 & Akyazı & Between Şerefiye and Reşadiye & 455 & $40^{\circ} 36^{\prime} 45^{\prime \prime} \mathrm{N}$ & $30^{\circ} 41^{\prime} 49^{\prime \prime} \mathrm{E}$ & 24.05.2017 \\
\hline 34 & Akyazı & Around of Şerefiye & 630 & $40^{\circ} 36^{\prime} 54^{\prime \prime} \mathrm{N}$ & $30^{\circ} 43^{\prime} 30^{\prime \prime} \mathrm{E}$ & 24.05.2017 \\
\hline 35 & Akyazı & Upper parts of Ballıkaya & 915 & $40^{\circ} 34^{\prime} 51^{\prime \prime} \mathrm{N}$ & $30^{\circ} 38^{\prime} 11^{\prime \prime} \mathrm{E}$ & 24.05.2017 \\
\hline 36 & Akyazı & Around of Hanyatak & 1140 & $40^{\circ} 33^{\prime} 21^{\prime \prime} \mathrm{N}$ & $30^{\circ} 36^{\prime} 05^{\prime \prime} \mathrm{E}$ & 24.05.2017 \\
\hline 37 & Akyazı & Around of Hanyatak & 1108 & $40^{\circ} 32^{\prime} 12^{\prime \prime} \mathrm{N}$ & $30^{\circ} 34^{\prime} 06^{\prime \prime} \mathrm{E}$ & 24.05.2017 \\
\hline 38 & Akyazı & $\begin{array}{l}\text { Between Hanyatak and } \\
\text { Acelle plateau }\end{array}$ & 1288 & $40^{\circ} 31^{\prime} 25^{\prime \prime} \mathrm{N}$ & $30^{\circ} 33^{\prime} 48^{\prime \prime} \mathrm{E}$ & 24.05.2017 \\
\hline 39 & Akyazı & Acelle plateau & 1159 & $40^{\circ} 30^{\prime} 42^{\prime \prime} \mathrm{N}$ & $30^{\circ} 37^{\prime} 27^{\prime \prime} \mathrm{E}$ & 24.05.2017 \\
\hline 40 & Kemaliye & The way of Kemaliye & 350 & $40^{\circ} 38^{\prime} 36^{\prime \prime} \mathrm{N}$ & $30^{\circ} 24^{\prime} 28^{\prime \prime} \mathrm{E}$ & 22.08 .2017 \\
\hline 41 & Arifiye & Between Kemaliye and Maksudiye & 450 & $40^{\circ} 38^{\prime} 21^{\prime \prime} \mathrm{N}$ & $30^{\circ} 24^{\prime} 17^{\prime \prime} \mathrm{E}$ & 22.08 .2017 \\
\hline 42 & Arifiye & Between Kemaliye and Maksudiye & 500 & $40^{\circ} 37^{\prime} 37^{\prime \prime} \mathrm{N}$ & $30^{\circ} 23^{\prime} 46^{\prime \prime} \mathrm{E}$ & 22.08.2017 \\
\hline 43 & Geyve & Between Maksudiye and Doğançay & 200 & $40^{\circ} 37^{\prime} 34^{\prime \prime} \mathrm{N}$ & $30^{\circ} 22^{\prime} 02^{\prime \prime} \mathrm{E}$ & 22.08.2017 \\
\hline 44 & Geyve & Between Celaller and Setçe & 1157 & $40^{\circ} 33^{\prime} 32^{\prime \prime} \mathrm{N}$ & $30^{\circ} 28^{\prime} 44^{\prime \prime} \mathrm{E}$ & 23.08.2017 \\
\hline 45 & Geyve & Between Celaller and Setçe & 1095 & $40^{\circ} 34^{\prime} 08^{\prime \prime} \mathrm{N}$ & $30^{\circ} 28^{\prime} 15^{\prime \prime} \mathrm{E}$ & 23.08.2017 \\
\hline 46 & Geyve & Around of Celaller & 1065 & $40^{\circ} 33^{\prime} 26^{\prime \prime} \mathrm{N}$ & $30^{\circ} 27^{\prime} 37^{\prime \prime} \mathrm{E}$ & 23.08.2017 \\
\hline[ & Geyve & Around of Celaller & 1155 & $40^{\circ} 33^{\prime} 32^{\prime \prime} \mathrm{N}$ & $30^{\circ} 26^{\prime} 33^{\prime \prime} \mathrm{E}$ & 23.08.2017 \\
\hline 48 & Geyve & Around of Setçe & 679 & $40^{\circ} 32^{\prime} 25^{\prime \prime} \mathrm{N}$ & $30^{\circ} 27^{\prime} 11 " \mathrm{E}$ & 23.08.2017 \\
\hline 49 & Geyve & Between Kulfallar and Kamışlı & 1168 & $40^{\circ} 33^{\prime} 03^{\prime \prime} \mathrm{N}$ & $30^{\circ} 23^{\prime} 44^{\prime \prime} \mathrm{E}$ & 23.08.2017 \\
\hline 50 & Geyve & Around of Kulfallar & 850 & $40^{\circ} 33^{\prime} 38^{\prime \prime} \mathrm{N}$ & $30^{\circ} 22^{\prime} 25^{\prime \prime} \mathrm{E}$ & 23.08.2017 \\
\hline 51 & rçek & $\begin{array}{l}\text { The way of Göktepedüzü } \\
\text { timber storage area }\end{array}$ & 835 & $40^{\circ} 35^{\prime} 36^{\prime \prime} \mathrm{N}$ & $30^{\circ} 32^{\prime} 36^{\prime \prime} \mathrm{E}$ & 24.08.2017 \\
\hline 52 & Karapürçek & Göktepedüzü timber storage area & 1005 & $40^{\circ} 35^{\prime} 45^{\prime \prime} \mathrm{N}$ & $30^{\circ} 32^{\prime} 37^{\prime \prime} \mathrm{E}$ & 24.08.2017 \\
\hline 53 & Karapürçek & $\begin{array}{l}\text { Around of Göktepedüzü } \\
\text { timber storage area }\end{array}$ & 1121 & $40^{\circ} 34^{\prime} 12^{\prime \prime} \mathrm{N}$ & $30^{\circ} 32^{\prime} 27^{\prime \prime} \mathrm{E}$ & 24.08.2017 \\
\hline 54 & Karapürçek & Around of Ahmediye & 750 & $40^{\circ} 35^{\prime} 10^{\prime \prime} \mathrm{N}$ & $30^{\circ} 33^{\prime} 11^{\prime \prime} \mathrm{E}$ & 24.08 .2 \\
\hline
\end{tabular}

The status of the taxa in Turkey were detected by reviewing the latest literatures and checklists [26-31]. In the bryofloristic list, only one herbarium number (e.g., ZNG 6030) for each taxon was given to avoid repetition. The voucher specimens were deposited in the bryophyte herbarium (ZNG) at Biology Department, Faculty of Science and Arts, Zonguldak Bülent Ecevit University. The nomenclature and arrangement of taxa was followed by the system proposed by the relevant literatures. [32, 33, 34]. In addition, the new records for A 2 grid-square and Turkey were determined by looking through to the correlated literatures [5-7, 30, 35, 36]. The life forms and preferred ecological features of the bryophytes in the study area such as; moisture conditions, light requirements and the $\mathrm{pH}$ of the substrates were estimated by using related studies and our field observations [37, 38, 39] and they are also given in the floristic list for each taxon. At the same time, the new records for the A2 grid square with (*), taxa recorded from Turkey for the second time with $(* *)$ in the bryofloristic list have been marked.

\section{Results}

In this study, a total of 193 taxa belonging to Bryophyta (32 families, 86 genera, 158 taxa), Marchantiophyta (22 families, 27 genera, 34 taxa), and Anthocerotophyta (1 family, 1 genus, 1 species) were identified. The bryofloristic list is given as below. 


\section{ANTHOCEROTOPHYTA}

Anthocerotaceae Dumort.

"Anthoceros caucasicus Steph. - Loc: 41; roadside slope on soil, ZNG 6027; Distribution in Turkey: A1, A4; CR (Italy, Spain). Ecological features: Acidophyte, hygrophyte, photophyte; Mat thalloid

MARCHANTIOPHYTA Stotler \& Crand.-Stotl.

Anastrophyllaceae L.Söderstr.

Barbilophozia barbata (Schreb.) Loeske - Loc: 25; on rocks, ZNG 6028; Distribution in Turkey: A2, A4, B6; CR (Ireland), EN (Netherlands), VU (Germany), NT (Italy). Ecological features: Subneutrophyte, hygrophyte, sciophyte; Mat smooth.

*Isopaches bicrenatus (Schmidel ex Hoffm.) H.Buch (Syn: Lophozia bicrenata (Schmidel) Dumort., Jungermannia bicrenata Schmidel ex Hoffm.) - Loc: 20; on wet soil, ZNG 6029; Distribution in Turkey: A1, A4; EN (Netherlands, Slovakia), VU (Canary Islands, Germany, Switzerland), NT (Italy, Czech Republic, Hungary). Ecological features: Acidophyte, mesophyte, photophyte; Mat smooth.

Cephaloziaceae Mig.

Fuscocephaloziopsis lunulifolia (Dumort.) Váňa et L.Söderstr. - Loc: 22; on decaying log, ZNG 6030; Distribution in Turkey: A2; NT (Italy). Ecological features: Acidophyte, hygrophyte, sciophyte; Mat smooth.

Cephaloziellaceae Douin

Cephaloziella divaricata (Sm.) Schiffn. - Loc: 19, 31, 52; on soil, road slope and decaying logs, ZNG 6031; Distribution in Turkey: A2; NT (Italy). Ecological features: Acidophyte, mesophyte, photophyte; Mat smooth.

Lophoziaceae Cavers

Lophozia ventricosa (Dicks.) Dumort. - Loc: 22; on decaying log, ZNG 6032; Distribution in Turkey: A1, A2, A4, C11; NT (Italy, Hungary), VU (Netherlands). Ecological features: Acidophyte, hygrophyte, photophyte; Mat smooth. Calypogeiaceae Arnell

Calypogeia fissa (L.) Raddi - Loc: 46, 50; on soil, ZNG 6033; Distribution in Turkey: A1, A2, A3, A4, B6, C12; EN (Slovakia, Bulgaria, Serbia, Latvia), NT (Finland, Italy, Sardinia, Czech Republic, Hungary). Ecological features: Acidophyte, hygrophyte, sciophyte; Mat smooth.

Lophocoleaceae Vanden Berghen

Chiloscyphus polyanthos (L.) Corda - Loc: 20; on soil near water resources, ZNG 6034; Distribution in Turkey: A1, A2, A4, A5, B6, B8, C11; VU (San Marino), NT (Italy, Sardinia). Ecological features: Acidophyte, hygrophyte, sciophyte; Mat smooth.

Lophocolea bidentata (L.) Dumort. - Loc: 46, 50; on soil, ZNG 6035; Distribution in Turkey: A1, A2, A3, A4, A5, B6, B7, C11, C12; VU (Finland), NT (Italy). Ecological features: Acidophyte, hygrophyte, sciophyte; Weft.

Lophocolea heterophylla (Schrad.) Dumort. - Loc: 9, 19, 23, 36; on decaying wood and soil, ZNG 6036; Distribution in Turkey: A1, A2, A3, A4, A5, B6, B7; NT (Italy). Ecological features: Acidophyte, hygrophyte, sciophyte; Mat smooth.

Lophocolea minor Nees - Loc: 17, 48; on soils, ZNG 6037; Distribution in Turkey: A1, A2, A3, A4, C13; CR (Netherlands), R (Latvia), NT (Italy, Germany). Ecological features: Subneutrophyte, mesophyte, sciophyte; Mat smooth.

Plagiochilaceae Müll.Frib

Plagiochila asplenioides (L.) Dumort. - Loc: 16; on rocks near stream, ZNG 6038; Distribution in Turkey: A1, A2, A3, A4, B6; EN (Netherlands), NT (Italy, Hungary), VU (Germany). Ecological features: Acidophyte, hygrophyte, sciophyte; Turf.

Plagiochila porelloides (Torrey ex Nees) Lindenb. - Loc: 14, 21, 36, 39, 41; on rocks and soil, ZNG 6039; Distribution in Turkey: A1, A2, A3, A4, B6, B7, C11, C12; CR (Netherlands), EN (Canary Islands), NT (Madeira). Ecological features: Subneutrophyte, mesophyte, sciophyte; Turf.

Scapaniaceae Mig.

Diplophyllum albicans (L.) Dumort. - Loc: 20, 52; on soil, ZNG 6040; Distribution in Turkey: A2, A4, A5; CR (Sardinia), EN (Hungary), VU (Netherlands), NT (Canary Islands, Italy). Ecological features: Acidophyte, hygrophyte, sciophyte; Mat smooth.

Scapania nemorea (L.) Grolle - Loc: 52; on soil, ZNG 6041; Distribution in Turkey: A2, A3, A4; EN (Netherlands), VU (Hungary, Estonia), NT (Finland, Italy, Germany). Ecological features: Acidophyte, hygrophyte, sciophyte; Weft.

Solenostomataceae Stotler et Crand.-Stotl.

Solenostoma gracillimum (Sm.) R.M.Schust. (Syn: Jungermannia gracillima Sm.) - Loc: 28, 50, 52, 54; forest road slope on soil, ZNG 6042; Distribution in Turkey: A2, A4, B6, C11. Ecological features: Subneutrophyte, hygrophyte, sciophyte; Mat smooth.

Frullaniaceae Lorch

Frullania dilatata (L.) Dumort. - Loc: 11, 12, 13, 14, 15, 16, 17, 21, 23, 24, 26, 31, 33, 35, 44, 45, 51, 53, common, ZNG 6043; Distribution in Turkey: A1, A2, A3, A4, A5, B6, C11, C12, C13; VU (Germany), NT (Italy). Ecological features: Acidophyte, hygrophyte, sciophyte; Mat smooth. 
Frullania tamarisci (L.) Dumort. - Loc: 15, 39; on tree trunks, ZNG 6044; Distribution in Turkey: A1, A2, A3, A4, B6, C12, C13; EN (Netherlands, Poland), VU (Austria, Germany, Estonia, Latvia), NT (Italy, Czech Republic, Switzerland, Hungary). Ecological features: Acidophyte, mesophyte, sciophyte; Mat smooth.

Lejeuneaceae Cavers

Cololejeunea rossettiana (C. Massal.) Schiffn. - Loc: 9, 14; on rocks, ZNG 6045; Distribution in Turkey: A1, A2, A4; CR (Luxembourg, Sardinia), EN (Austria), VU (Czech Republic, Slovakia, Switzerland, Serbia), R (Germany, Poland, Ukrania), NT (Italy, Hungary). Ecological features: Basiphyte, hygrophyte, sciophyte; Mat rough.

Lejeunea cavifolia (Ehrh.) Lindb. - Loc: 4, 34; on soil, ZNG 6046; Distribution in Turkey: A1, A2, A3, A4, B6, B7, C11; RE (Netherlands), VU (Latvia), NT (Italy, Germany, Hungary). Ecological features: Acidophyte, hygrophyte, sciophyte; Mat smooth.

Porellaceae Cavers

Porella platyphylla (L.) Pfeiff. - Loc: 7, 9, 11, 12, 13, 15, 16, 17, 21, 23, 24, 27, 29, 32, 35, 39, 44, 45, 48, 53; on soil, rocks and tree trunks, ZNG 6047; Distribution in Turkey: A1, A2, A3, A4, A5, B6, B7, B8, C11, C12, C13; EN (Poland), NT (Finland, Italy, Estonia). Ecological features: Acidophyte, mesophyte, sciophyte; Fan.

Radulaceae Müll.Frib.

Radula complanata (L.) Dumort. - Loc: 11, 13, 14, 15, 16, 17, 20, 21, 23, 24, 26, 27, 29, 32, 33, 35, 39, 40, 53; on soil and tree trunks, ZNG 6048; Distribution in Turkey: A1, A2, A3, A4, A5, B6, B7, C11, C12, C13; VU (Germany), NT (Italy). Ecological features: Acidophyte, hygrophyte, sciophyte; Mat smooth.

Radula lindenbergiana Gottsche ex C.Hartm. - Loc: 14, 15, 51; on tree trunks, ZNG 6049; Distribution in Turkey: A1, A2, A3, A4, B6, C11; VU (Finland, Czech Republic, Bulgaria), NT (Italy). Ecological features: Acidophyte, hygrophyte, sciophyte; Mat smooth.

METZGERIALES Chalaud

Aneuraceae H.Klinggr.

Aneura pinguis (L.) Dumort. - Loc: 48, 54; on wet soil in water resources, ZNG 6050; Distribution in Turkey: A1, A2, A4, C11, C12, C13; VU (Czech Republic), NT (Italy, Germany, Hungary). Ecological features: Subneutrophyte, hygrophyte, sciophyte; Mat thalloid.

Riccardia palmata (Hedw.) Carruth. - Loc: 51; on wet tree trunks near stream, ZNG 6051; Distribution in Turkey: A2, A4; NT (Finland, Hungary, Italy), RE (Sicily). Ecological features: Acidophyte, hygrophyte, sciophyte; Mat thalloid.

Metzgeriaceae H.Klinggr.

Metzgeria conjugata Lindb. - Loc: 17, 43, 51; on rocks and soil, ZNG 6052; Distribution in Turkey: A1, A2, A3, A4, A5, B6, B7, C11, C12, C13; RE (Netherlands), CR (Estonia), VU (Canary Islands, Poland), NT (Sweden, Italy, Hungary). Ecological features: Subneutrophyte, hygrophyte, sciophyte; Mat thalloid.

Metzgeria furcata (L.) Dumort - Loc: 11, 13, 14, 18, 26, 36, 37, 40, 50, 51; on tree trunks, ZNG 6053; Distribution in Turkey: A1, A2, A3, A4, A5, B6, B7, C11, C12, C13; VU (Latvia), NT (Italy, Germany). Ecological features: Acidophyte, mesophyte, sciophyte; Mat thalloid.

FOSSOMBRONIALES Schljakov

Fossombroniaceae Hazsl.

Fossombronia angulosa (Dicks.) Raddi - Loc: 4, 40; on soil, ZNG 6054; Distribution in Turkey: A1, A2, A4, B6, C11, C12; RE (Czech Republic), EN (Bulgaria), VU (Switzerland), NT (Italy). Ecological features: Acidophyte, hygrophyte, sciophyte; Mat smooth.

PELLIALES He-Nygren, Juslen, Ahonen, Glenny \& Piippo

Pelliaceae H.Klinggr.

Pellia endiviifolia (Dicks.) Dumort - Loc: 2, 20, 41, 54; on wet rocks and soil in water resources, ZNG 6055; Distribution in Turkey: A1, A2, A3, A4, A5, B6, B7, B9, C11, C12; NT (Finland, Italy, San Marino). Ecological features: Subneutrophyte, hygrophyte, sciophyte; Mat thalloid.

MARCHANTIOPSIDA Gonquist, Takht \& W. Zimm.

BLASIALES Stotler et Crand.-Stotl.

Blasiaceae H.Klinggr.

Blasia pusilla L. - Loc: 41, 54; forest road slope on wet sandy soil and rocks, ZNG 6056; Distribution in Turkey: A1, A2, A3, A4; EN (Luxembourg, Hungary), VU (Portugal, Spain, Netherlands, Switzerland), NT (Italy, Germany). Ecological features: Basiphyte, hygrophyte, photophyte; Mat thalloid.

LUNULARIALES H.Klinggr.

Lunulariaceae H.Klinggr.

Lunularia cruciata (L.) Lindb - Loc: 4, 41; road slope on soil, ZNG 6057; Distribution in Turkey: A1, A2, A3, A4, A5, B6, B7, B9, C11, C12, C13; CR (Romania, Slovenia), NT (Italy, San Marino, Luxembourg). Ecological features: Subneutrophyte, mesophyte, sciophyte; Mat thalloid.

MARCHANTIALES Limpr

Conocephalaceae Müll.Frib. ex Grolle 
Conocephalum conicum (L.) Dumort. - Loc: 16, 30, 41, 51, 54; on wet rocks and soil near stream bed, ZNG 6058; Distribution in Turkey: A1, A2, A3, A4, A5, B6, B8, C11, C12, C13; EN (Finland, Canary Islands), VU (Netherlands), NT (Italy, San Marino). Ecological features: Basiphyte, hygrophyte, sciophyte; Mat thalloid.

Marchantiaceae Lindl.

*Marchantia polymorpha subsp. montivagans Bischl. \& Boisselier - Loc: 20; on soil in water resources, ZNG 6059; Distribution in Turkey: A1, B6, B9, C13; EN (Ireland). Ecological features: Acidophyte, hygrophyte, sciophyte; Mat thalloid.

Marchantia polymorpha Bischl.\& Boisselier subsp. polymorpha - Loc: 2, 20, 41, 51; on soil in water resources, ZNG 6060; Distribution in Turkey: A1, A2, A3, A4, A5, B6, B7, B8, B9, C11, C12, C13, C15; VU (Slovenia). Ecological features: Acidophyte, hygrophyte, sciophyte; Mat thalloid.

Ricciaceae Rchb.

*Riccia sorocarpa Bisch. - Loc: 21, 44; on soil, ZNG 6061; Distribution in Turkey: A1, A5, B6, B8, C11, C12; NT (Italy, Spain). Ecological features: Acidophyte, mesophyte, photophyte; Solitary thalloid.

\section{BRYOPHYTA Schimp.}

Sphagnaceae Dumort.

**Sphagnum contortum Schultz - Loc: 20; peatlands on soil, ZNG 6062; Distribution in Turkey: A4; NT (Finland, Czech Republic), VU (Spain, Slovakia, Hungary, Montenegro, Serbia). Ecological features: Acidophyte, hygrophyte, photophyte; Turf.

Polytrichaceae Schwägr.

Atrichum angustatum (Brid.) Bruch \& Schimp. - Loc: 35; on soil, ZNG 6063; Distribution in Turkey: A1, A2, A4; RE (Ireland, Northern Ireland, Belgium), CR (Sweden, Great Britain), EN (Czech Republic), VU (Germany, Estonia, Lithuania). Ecological features: Acidophyte, hygrophyte, sciophyte; Turf.

Pogonatum aloides (Hedw.) P. Beauv. - Loc: 42; on soil, ZNG 6064; Distribution in Turkey: A1, A2, A3, A4, B6; EN (Estonia), R (Lithuania). Ecological features: Acidophyte, mesophyte, sciophyte; Protonemal turf.

Pogonatum urnigerum (Hedw.) P. Beauv. - Loc: 2; on soil, ZNG 6065; Distribution in Turkey: A1, A2, A3, A4, B6, C11; VU (Netherlands). Ecological features: Acidophyte, mesophyte, photophyte; Turf.

Polytrichum commune Hedw. - Loc: 20; peatlands on soil, ZNG 6066; Distribution in Turkey: A1, A2, A3, A4, B6; VU (Canary Islands, Hungary), NT (Sicily). Ecological features: Acidophyte, hygrophyte, sciophyte; Turf.

Polytrichum formosum Hedw. - Loc: 39, 49; on soil, ZNG 6067; Distribution in Turkey: A1, A2, A3, A4, A5;

VU (Canary Islands). Ecological features: Acidophyte, mesophyte, sciophyte; Turf.

Polytrichum juniperinum Hedw. - Loc: 20, 21; on soil, ZNG 6068; Distribution in Turkey: A1, A2, A3, A4,

A5, B6, B7, B8, B10, C11, C13. Ecological features: Acidophyte, kserofit, photophyte; Turf.

Polytrichum piliferum Hedw. - Loc: 44; on soil, ZNG 6069; Distribution in Turkey: A1, A2, A4, B6, B7, B8,

C11; NT (Sicily). Ecological features: Acidophyte, kserofit, photophyte; Turf.

Tetraphidaceae Schimp.

Tetraphis pellucida Hedw. - Loc: 22, 53; on decaying logs and stumps, ZNG 6070; Distribution in Turkey: A1, A2, A4; VU (Montenegro, Serbia). Ecological features: Acidophyte, hygrophyte, sciophyte; Turf.

Diphysciaceae M.Fleisch.

Diphyscium foliosum (Hedw.) D. Mohr - Loc: 44, 52; on soil, ZNG 6071; Distribution in Turkey: A2, A4; EN (Netherlands), VU (Estonia), NT (Finland, Germany, Hungary). Ecological features: Acidophyte, hygrophyte, sciophyte; Turf.

Encalyptaceae Schimp.

Encalypta streptocarpa Hedw. - Loc: 14, 23, 28, 43; on rocks crevices, ZNG 6072; Distribution in Turkey: A1, A2, A3, A4, B5, B6, B7, C11, C12, C13; VU (Canary Islands), NT (Sicily). Ecological features: Subneutrophyte, hygrophyte, photophyte; Tuft

FUNARIALES M.Fleisch.

Funariaceae Schwägr.

*Entosthodon pulchellus (H. Philib.) Brugués - Loc: 10; on soil, ZNG 6073; Distribution in Turkey: B6, B9, B10, C11, C12, C13; CR (Switzerland), NT (Great Britain, Hungary), EN (Ireland, Czech Republic). Ecological features: Acidophyte, hygrophyte, photophyte; Turf.

Funaria hygrometrica Hedw. - Loc: 36, 42; on soil, ZNG 6074; Distribution in Turkey: A1, A2, A3, A4, A5,

B6, B7, B8, B10, C11, C12, C13, C14, C15. Ecological features: Subneutrophyte, hygrophyte, photophyte; Tuft.

Grimmiaceae Arn.

*Grimmia nutans Bruch - Loc: 14; on moist rocks, ZNG 6075; Distribution in Turkey: A4, B6, B8, C11, C13;

VU (Canary Islands). Ecological features: Acidophyte, kserofit, photophyte; Cushion.

Grimmia pulvinata (Hedw.) Sm. - Loc: 8; on moist rocks, ZNG 6076; Distribution in Turkey: A1, A2, A3, A4, A5, B6, B7, B8, B9, B10, C11, C12, C13, C14. Ecological features: Acidophyte, kserofit, photophyte; Cushion. 
Racomitrium affine (F. Weber \& D. Mohr) Lindb. - Loc: 14; on soil, ZNG 6077; Distribution in Turkey: A1, A2, A4; VU (Luxembourg, Bulgaria), NT (Slovakia). Ecological features: Acidophyte, hygrophyte, sciophyte; Turf.

Racomitrium canescens (Hedw.) Brid. - Loc: 26; on soil, ZNG 6078; Distribution in Turkey: A1, A2, A3, A4; VU (Luxembourg, Bulgaria), NT (Slovakia). Ecological features: Acidophyte, mesophyte, photophyte; Turf.

Racomitrium elongatum Ehrh. ex Frisvoll - Loc: 20; on soil, ZNG 6079; Distribution in Turkey: A1, A2, A4;

VU (Ireland, Northern Ireland, Netherlands). Ecological features: Acidophyte, mesophyte, photophyte; Turf.

Schistidium apocarpum (Hedw.) Bruch \& Schimp. - Loc: 23; on rocks, ZNG 6080; Distribution in Turkey: A1, A2, A3, A4, B5, B6, B7, B8, B9, C11, C12, C13, C14; VU (Canary Islands). Ecological features: Acidophyte, hygrophyte, photophyte; Tuft

Schistidium crassipilum H.H. Blom - Loc: 23; on rocks, ZNG 6081; Distribution in Turkey: A1, A2, A4, B6, C13; NT (Finland). Ecological features: Basiphyte, mesophyte, photophyte; Turf.

*Schistidium papillosum Culm. - Loc: 13; on rocks, ZNG 6082; Distribution in Turkey: A4; NT (Great Britain), EN (Hungary). Ecological features: Subneutrophyte, kserofit, photophyte; Cushion.

*Schistidium robustum (Nees \& Hornsch.) H.H. Blom - Loc: 13; on moist rocks, ZNG 6083; Distribution in Turkey: A1; VU (Ireland), CR (Romania, Luxemburg), NT (Hungary). Ecological features: Subneutrophyte, kserofit, photophyte; Cushion.

Fissidentaceae Schimp.

Fissidens dubius P. Beauv. - Loc: 43; on shaded rocks, ZNG 6084; Distribution in Turkey: A1, A2, A4, B6, C11, C12, C13. Ecological features: Subneutrophyte, mesophyte, sciophyte; Turf.

Fissidens gracilifolius Brugg.-Nann. \& Nyholm - Loc: 53; on soil, ZNG 6085; Distribution in Turkey: A2, A4; RE (Finland), VU (Norway, Bulgaria, Romania). Ecological features: Subneutrophyte, mesophyte, sciophyte; Turf.

Fissidens rivularis (Spruce) Schimp. - Loc: 54; on wet rocks in stream bed, ZNG 6086; Distribution in Turkey: A1, A2, B6, C13; CR (Romania), VU (Ireland, Switzerland, Bulgaria), R (Germany). Ecological features: Acidophyte, rheophyte, sciophyte; Turf.

Fissidens taxifolius Hedw. - Loc: 1, 11, 15, 16, 29, 30, 32, 40, 45; on soil, ZNG 6087; Distribution in Turkey: A1, A2, A3, A4, B6, C11, C12, C13. Subneutrophyte, mesophyte, sciophyte; Turf.

Ditrichaceae Limpr.

Ceratodon purpureus (Hedw.) Brid. - Loc: 8, 18, 20, 35, 40, 44, 47; on soil and stump, ZNG 6088; Distribution in Turkey: A1, A2, A3, A4, A5, B6, B7, B8, B10, C11, C13, C14. Ecological features: Acidophyte, mesophyte, photophyte; Turf.

Ditrichum heteromallum (Hedw.) E.Britton - Loc: 41, 45; forest roadside slopes on soil, ZNG 6089; Distribution in Turkey: A1, A2, A3, A4, B6, B7. VU (Netherlands), NT (Germany, Luxembourg, Hungary). Ecological features: Acidophyte, mesophyte, sciophyte; Tuft.

Ditrichum pallidum (Hedw.) Hampe - Loc: 34; on soil, ZNG 6090; Distribution in Turkey: A1, A2, A3, A4; RE (Sweden), EN (Switzerland, Bulgaria), VU (Czech Republic, Germany, Estonia), NT (Romania). Ecological features: Acidophyte, mesophyte, sciophyte; Tuft.

Rhabdoweisiaceae Limpr

Dichodontium pellucidum (Hedw.) Schimp. - Loc: 15, 54; forest roadside slopes on wet soil and rocks, ZNG 6091; Distribution in Turkey: A1, A2, A3, A4, B6; NT (Hungary, Estonia). Ecological features: Acidophyte, hygrophyte, photophyte; Cushion.

Dicranaceae Schimp.

Dicranella heteromalla (Hedw.) Schimp. - Loc: 20; on soil, ZNG 6092; Distribution in Turkey: A1, A2, A3, A4, A5, C11, C13. Ecological features: Subneutrophyte, mesophyte, sciophyte; Turf.

Dicranella howei Ren. \& Card. - Loc: 33; on soil, ZNG 6093; Distribution in Turkey: A1, A2, A3, A4, C11, C12, C13; VU (Switzerland), NT (Hungary). Ecological features: Basiphyte, kserofit, photophyte; Turf.

Dicranella rufescens (With.) Schimp. - Loc: 54; on soil, ZNG 6094; Distribution in Turkey: A1, A2, A4; RE (Estonia), VU (Netherlands, Switzerland, Bulgaria, Romania), NT (Slovakia, Hungary). Ecological features: Acidophyte, hygrophyte, photophyte; Turf.

Dicranella varia (Hedw.) Schimp. - Loc: 33, 36, 47; on soil, ZNG 6095; Distribution in Turkey: A1, A2, A3, A4, B6, B7, C11, C12, C13. Ecological features: Basiphyte, hygrophyte, photophyte; Turf.

Dicranum scoparium Hedw. - Loc: 9, 19, 20, 27, 37; on soil and decaying log, ZNG 6096; Distribution in Turkey: A1, A2, A3, A4, A5, B6, B7, B8, C11; VU (Canary Islands), NT (Sicily). Ecological features: Acidophyte, hygrophyte, sciophyte; Tuft.

Dicranum tauricum Sapjegin - Loc: 18, 35; on decaying log and stump, ZNG 6097; Distribution in Turkey: A1, A2, A3, A4, B6, B7; VU (Romania), NT (Finland). Ecological features: Acidophyte, mesophyte, sciophyte; Tuft.

Pottiaceae Schimp.

Barbula convoluta Hedw. - Loc: 35, 39; on soil, ZNG 6098; Distribution in Turkey: A1, A2, A3, A4, B6, B7, C11, C12, C13. Ecological features: Subneutrophyte, mesophyte, photophyte; Turf.

Barbula unguiculata Hedw. - Loc: 3, 53; on soil, ZNG 6099; Distribution in Turkey: A1, A2, A3, A4, B6, B7, B8, B10, C11, C12, C13. Ecological features: Acidophyte, hygrophyte, photophyte; Turf. 
Didymodon luridus Hornsch. - Loc: 33; on soil, ZNG 6100; Distribution in Turkey: A1, A2, A4, B6, C11, C12, C13, C15; RE (Sweden), EN (Austria), NT (Czech Republic). Ecological features: Basiphyte, kserofit, photophyte; Turf. Didymodon rigidulus Hedw. - Loc: 23; on soil covered rocks, ZNG 6101; Distribution in Turkey: A1, A2, A4, B6, B8, C11, C13. Ecological features: Subneutrophyte, kserofit, sciophyte; Tuft.

Didymodon vinealis (Brid.) R.H. Zander - Loc: 7, 43; on soil, ZNG 6102; Distribution in Turkey: A1, A2, A3, A4, B6, B7, B8, B9, B10, C11, C12, C13, C14; CR (Switzerland), EN (Austria, Czech Republic), VU (Sweden, Estonia), NT (Germany). Ecological features: Subneutrophyte, kserofit, photophyte; Tuft.

*Ephemerum crassinervium subsp. sessile (Bruch) Holyoak - Loc: 2; on soil, ZNG 6103; Distribution in Turkey: A1, C11, C12; EN (Germany). Ecological features: Subneutrophyte, kserofit, photophyte; Protonemal turf.

Leptobarbula berica (De Not.) Schimp. - Loc: 28; on rocks, ZNG 6104; Distribution in Turkey: A1, A2, B6, C11; VU (Luxembourg). Ecological features: Subneutrophyte, kserofit, sciophyte; Turf.

Syntrichia calcicola J.J. Amann - Loc: 52; on soil, ZNG 6105; Distribution in Turkey: A1, A2, B6, B8, B9, C11, C13; EN (Slovakia), NT (Sicily). Ecological features: Acidophyte, hygrophyte, sciophyte; Turf.

Syntrichia papillosa (Wilson) Jur. - Loc: 11; on tree trunk, ZNG 6106; Distribution in Turkey: A1, A2, A4; EN (Bulgaria), VU (Slovakia), R (Poland). Ecological features: Subneutrophyte, mesophyte, sciophyte; Tuft.

Syntrichia princeps (De Not.) Mitt. - Loc: 26, 27, 44; on tree trunk, ZNG 6107; Distribution in Turkey: A1, A2, A4, B6, B7, B8, B10, C11, C12, C13, C14, C15; NT (Sweden, Great Britain), RE (Ireland, Northern Ireland), R (Germany), EN (Luxembourg), VU (Bulgaria), CR (Romania). Ecological features: Subneutrophyte, mesophyte, sciophyte; Turf.

Syntrichia ruralis (Hedw.) F. Weber \& D. Mohr var. ruralis - Loc: 10, 14, 15, 26, 29, 38, 39; on rocks and tree trunk, ZNG 6108; Distribution in Turkey: A1, A2, A3, A4, A5, B6, B7, B8, B9, B10, C11, C12, C13, C14, C15. Ecological features: Subneutrophyte, kserofit, photophyte; Turf.

Syntrichia ruralis (Hedw.) F. Weber \& D. Mohr var. ruraliformis (Besch.) Delogne - Loc: 21; on rocks and soil, ZNG 6109; Distribution in Turkey: A1, A2, A3, A4, A5, B6, B7, B8, B9, B10, C11, C12, C13, C14, C15; EN (Austria), VU (Romania). Ecological features: Subneutrophyte, kserofit, photophyte; Turf.

Syntrichia virescens (De Not.) Ochyra - Loc: 53; on tree trunk, ZNG 6110; Distribution in Turkey: A1, A2, A4, A5, B6, B10, C11, C13; VU (Norway, Bulgaria), R (Poland). Ecological features: Subneutrophyte, mesofit, sciophyte; Turf.

Tortella squarrosa (Brid.) Limpr. (Syn: Pleurochaete squarrosa (Brid.) Lindb.) - Loc: 11; on soil, ZNG 6111; Distribution in Turkey: A1, A2, A3, A4, B6, C11, C12, C13; RE (Sweden), EN (Austria), VU (Germany, Switzerland), R (Poland), NT (Ireland, Czech Republic, Slovakia, Romania). Ecological features: Basiphyte, kserofit, photophyte; Turf.

Tortella tortuosa (Hedw.) Limpr. - Loc: 3, 8, 13, 23; on soil and rock crevices, ZNG 6112; Distribution in

Turkey: A1, A2, A3, A4, A5, B6, B7, B8, C11, C12, C13. Ecological features: Basiphyte, mesophyte, sciophyte; Tuft.

Tortula muralis Hedw. - Loc: 13; on rock, ZNG 6113; Distribution in Turkey: A1, A2, A3, A4,B5, B6, B7,

B8, B9, B10, C11, C12, C13, C14, C15. Ecological features: Subneutrophyte, mesophyte, sciophyte; Turf.

Tortula schimperi M.J. Cano, O. Werner \& J. Guerra - Loc: 16, 36; on soil, ZNG 6114; Distribution in Turkey:

A1, A2, A4, B6, B7, B8, C11; NT (Romania). Ecological features: Subneutrophyte, mesophyte, sciophyte; Tuft.

Weissia controversa Hedw. - Loc: 4, 32, 33, 52; on soil, ZNG 6115; Distribution in Turkey: A1, A2, A3, A4,

B6, B7, B8, C11, C12, C13; VU (Netherlands). Ecological features: Acidophyte, kserofit, photophyte; Turf.

Bryaceae Schwägr.

Bryum argenteum Hedw. - Loc: 3; on soil, ZNG 6116; Distribution in Turkey: A1, A2, A3, A4, A5, B6, B7, B8, B9, B10, C11, C12, C13, C14. Ecological features: Subneutrophyte, mesophyte, photophyte; Turf.

Bryum dichotomum Hedw. - Loc: 2; on soil, ZNG 6117; Distribution in Turkey: A1, A2, A4, B6, B8, B10,

C11, C12, C13; VU (Estonia), NT (Romania). Ecological features: Subneutrophyte, kserofit, photophyte; Turf.

Bryum turbinatum (Hedw.) Turn. - Loc: 24; waterside on wet soil, ZNG 6118; Distribution in Turkey: A1, A2, A4, B6, B7, B8, C11, C13, C15. NT (Finland, Romania), VU (Norway, Sweden, Slovakia), RE (Great Britain, Ireland, Belgium, Netherlands), EN (Czech Republic). Ecological features: Acidophyte, mesophyte, sciophyte; Turf.

Imbribryum alpinum (Huds. ex With.) N. Pedersen (Syn: (Syn: Bryum alpinum Huds. ex With.) - Loc: 20, 25; wetland area on soil, ZNG 6119; Distribution in Turkey: A1, A2, A3, A4, B6, B7, B8, B9, C11, C12, C13, C15; VU (Germany, Luxembourg), NT (Czech Republic, Switzerland). Ecological features: Acidophyte, hygrophyte, photophyte; Turf.

Ptychostomum capillare (Hedw.) Holyoak \& N. Pedersen (Syn: Bryum capillare Hedw.) - Loc: 14, 49; on rocks and soil, ZNG 6120; Distribution in Turkey: A1, A2, A3, A4, A5, B6, B7, B8, B9, B10, C11, C12, C13. Ecological features: Subneutrophyte, mesophyte, sciophyte; Turf.

Ptychostomum imbricatulum (Müller Hal.) D. T. Holyoak \& N. Pedersen (Syn: Bryum caespiticium Hedw.) Loc: 13; on rock crevices, ZNG 6121; Distribution in Turkey: A1, A2, A3, A4, B6, B7, B8, B9, B10, C11, C12, C13, C14, C15; VU (Ireland, Northern Ireland). Ecological features: Subneutrophyte, mesophyte, photophyte; Turf.

Ptychostomum moravicum (Podp.) Ros \& Mazimpaka (Syn: Bryum moravicum Podp., B. flaccidum auct. non Brid., B. laevifilum Syed, B. subelegans auct. non Kindb.) - Loc: 15, 17, 19, 21, 23, 26, 29, 39, 43; on soil, tree trunks 
and decaying logs, ZNG 6122; Distribution in Turkey: A1, A2, A3, A4, B6, B7, B8, C13. Ecological features: Acidophyte, hygrophyte, sciophyte; Turf.

Ptychostomum pallens (Sw.) J.R.Spence (Syn: Bryum pallens (Brid.) Sw.) - Loc: 2, 47; waterside on wet soil, ZNG 6123; Distribution in Turkey: A1, A2, A3, A4, B6, B10, C11, C12, C13; EN (Canary Islands), VU (Hungary). Ecological features: Acidophyte, hygrophyte, sciophyte; Turf.

Ptychostomum pseudotriquetrum (Hedw.) J.R. Spence \& H.P. Ramsay (Syn: Bryum pseudotriquetrum (Hedw.) P.Gaertn. et al.) - Loc: 8, 20; waterside on soil, ZNG 6124; Distribution in Turkey: A1, A2, A3, A4, B6, B7, B8, B9, B10, C11, C12, C13; VU (Canary Islands). Ecological features: Subneutrophyte, hygrophyte, sciophyte; Turf.

Mniaceae Schwägr.

Epipterygium tozeri (Grev.) Lindb. - Loc: 2, 7, 30, 41; on soil, ZNG 6125; Distribution in Turkey: A1, A2, A4, B6, C11, C13; VU (Switzerland). Ecological features: Acidophyte, hygrophyte, sciophyte; Turf scattered.

Mnium marginatum (Dicks.) P. Beauv. - Loc: 14, 23; on rock crevices soil, ZNG 6126; Distribution in Turkey: A1, A2, A3, A4, C13; VU (Netherlands), NT (Germany). Ecological features: Subneutrophyte, mesophyte, sciophyte; Turf.

Mnium spinulosum Bruch \& Schimp. - Loc: 19; on soil, ZNG 6127; Distribution in Turkey: A2; NT (Spain). Ecological features: Acidophyte, mesophyte, sciophyte; Turf.

Mnium stellare Hedw. - Loc: 16; on soil, ZNG 6128; Distribution in Turkey: A1, A2, A3, A4, B6, B7; VU (Netherlands). Ecological features: Subneutrophyte, hygrophyte, sciophyte; Turf.

Plagiomnium affine (Blandow ex Funck) T.J. Kop. - Loc: 9, 11, 31; on soil, ZNG 6129; Distribution in Turkey: A1, A2, A3, A4, B6, B8. Ecological features: Acidophyte, hygrophyte, sciophyte; Mat smooth.

Plagiomnium cuspidatum (Hedw.) T.J. Kop. - Loc: 23; on soil, ZNG 6130; Distribution in Turkey: A1, A2, A3, A4, B6; NT (Ireland, Northern Ireland). Ecological features: Subneutrophyte, hygrophyte, sciophyte; Mat smooth.

Plagiomnium undulatum (Hedw.) T.J. Kop. - Loc: 15; on rocks, ZNG 6131; Distribution in Turkey: A1, A2, A3, A4, B6, B7, C11, C13. Ecological features: Acidophyte, hygrophyte, sciophyte; Turf.

Pohlia cruda (Hedw.) Lindb. - Loc: 37; on soil, ZNG 6132; Distribution in Turkey: A1, A2, A3, A4, A5, B6,

B7, B8, C11, C13; VU (Canary Islands). Ecological features: Acidophyte, mesophyte, sciophyte; Tuft.

Pohlia melanodon (Brid.) A.J. Shaw - Loc: 30, 54; on soil, ZNG 6133; Distribution in Turkey: A1, A2, A4, B6, B7, C10, C11, C13; VU (Czech Republic, Slovakia), NT (Finland, Switzerland). Ecological features: Subneutrophyte, hygrophyte, sciophyte; Turf.

Pohlia wahlenbergii (F. Weber \& D. Mohr) A.L. Andrews - Loc: 47; on soil, ZNG 6134; Distribution in Turkey: A1, A2, A3, A4, B6, B8, B10, C11, C12; VU (Canary Islands). Ecological features: Acidophyte, hygrophyte, photophyte; Turf.

Rhizomnium punctatum (Hedw.) T.J. Kop. - Loc: 14; on wet soil, ZNG 6135; Distribution in Turkey: A1, A2, A3, A4, B6, B7, B8; NT (Sicily). Ecological features: Acidophyte, hygrophyte, sciophyte; Turf.

Bartramiaceae Schwägr.

Bartramia pomiformis Hedw. - Loc: 30; on soil, ZNG 6136; Distribution in Turkey: A1, A2, A4, B6, C11; EN (Netherlands), VU (Estonia, Latvia), NT (Canary Islands, Germany). Ecological features: Acidophyte, hygrophyte, sciophyte; Tuft.

Philonotis arnelli Husn. - Loc: 2, 12; on wet soil, ZNG 6137; Distribution in Turkey: A1, A2, A4. Ecological features: Acidophyte, hygrophyte, sciophyte; Turf.

Philonotis caespitosa Jur. - Loc: 20, 24, 48; water resources on wet soil, ZNG 6138; Distribution in Turkey: A1, A2, A4, B6, B8, C11, C13; EN (Slovakia, Hungary), VU (Luxembourg, Switzerland, Bulgaria, Slovenia), R (Poland, Lithuania), NT (Ireland, Northern Ireland, Germany, Estonia). Ecological features: Acidophyte, hygrophyte, photophyte; Turf.

Philonotis calcarea (Bruch \& Schimp.) Schimp. - Loc: 24; water resources on wet soil, ZNG 6139; Distribution in Turkey: A1, A2, A3, A4, A5, B6, B7, B9, B10, C11, C12, C13; CR (Luxembourg), EN (Finland, Canary Islands), VU (Germany), NT (Sweden, Sicily). Ecological features: Basiphyte, hygrophyte, photophyte; Turf.

Philonotis capillaris Lindb. - Loc: 20; water resources on wet soil, ZNG 6140; Distribution in Turkey: A1, A2, A4, C11, C13; RE (Netherlands), EN (Ireland, Northern Ireland, Austria, Czech Republic, Romania), VU (Germany, Poland, Switzerland, Bulgaria), NT (Finland, Sicily, Luxembourg, Hungary, Slovenia). Ecological features: Acidophyte, hygrophyte, sciophyte; Turf.

Philonotis fontana (Hedw.) Brid. - Loc: 2, 20, 25; water resources on wet soil, ZNG 6141; Distribution in Turkey: A1, A2, A3, A4, B6, B7, B8, B9, B10, C13; VU (Canary Islands, Hungary), NT (Germany). Ecological features: Acidophyte, hygrophyte, sciophyte; Turf.

Orthotrichaceae Arn.

Lewinskya affinis (Schrad. ex Brid.) F.Lara, Garilleti \& Goffinet (Syn: Orthotrichum affine Schrad. ex Brid.) - Loc: 5, 11, 14, 17, 24, 27, 29, 39, 44; on tree trunk, ZNG 6142; Distribution in Turkey: A1, A2, A3, A4, B6, B7, B8, B10, C11, C13. Ecological features: Acidophyte, kserofit, sciophyte; Cushion. 
Lewinskya speciosa (Nees) F.Lara, Garilleti \& Goffinet (Syn. Orthotrichum speciosum Nees) - Loc: 10, 11, 13, 14, 23, 45; on tree trunk, ZNG 6143; Distribution in Turkey: A1, A2, B6, B7, B8, C11, C13; VU (Ireland), NT (Great Britain, Sicily, Slovakia). Ecological features: Subneutrophyte, mesophyte, sciophyte; Cushion.

Lewinskya striata (Hedw.) F.Lara, Garilleti \& Goffinet (Syn: Orthotrichum striatum Hedw.) - Loc: 11, 13, 14, 15, 17, 21, 26, 29, 32, 45; on tree trunk, ZNG 6144; Distribution in Turkey: A1, A2, A3, A4, B6, B7, B8, C11, C13; EN (Finland), VU (Slovakia), R (Latvia, Lithuania), NT (Germany). Ecological features: Basiphyte, mesophyte, photophyte; Cushion.

Orthotrichum anomalum Hedw. - Loc: 21, 38; on rock, ZNG 6145; Distribution in Turkey: A1, A2, A3, A4, B6, B7, B8, B10, C11, C12, C13, C14. Ecological features: Acidophyte, kserofit, photophyte; Cushion.

Orthotrichum cupulatum Hoffm. ex Brid. - Loc: 14, 25; on rock, ZNG 6146; Distribution in Turkey: A1, A2, A5, B6, B7, B8, B9, B10, C11, C12, C13, C14, C15; NT (Germany). Ecological features: Subneutrophyte, hygrophyte, sciophyte; Cushion.

Orthotrichum diaphanum Brid. - Loc: 32, 43, 44; on tree trunk, ZNG 6147; Distribution in Turkey: A1, A2, A3, A4, B6, B7, B8, C11, C12, C13. Ecological features: Subneutrophyte, kserofit, photophyte; Cushion.

Orthotrichum pallens Bruch ex Brid. - Loc: 14, 15, 17, 23, 32, 35, 44; on tree trunk, ZNG 6148; Distribution in Turkey: A1, A2, A3, B6, B7, B8, C11, C12, C13; EN (Great Britain, Ireland), NT (Sweden, Luxembourg, Slovakia). Ecological features: Subneutrophyte, hygrophyte, sciophyte; Cushion.

Orthotrichum pumilum Sw. ex anon. - Loc: 11, 12, 17, 18, 21, 23, 24, 39; on tree trunk, ZNG 6149; Distribution in Turkey: A1, A2, A3, A4, B6, B7, B8, C12, C13; EN (Great Britain), VU (Canary Islands), NT (Bulgary, Hungary). Ecological features: Subneutrophyte, kserofit, photophyte; Cushion.

Orthotrichum stramineum Hornsch. ex Brid. - Loc: 26, 35, 39, 45; on tree trunk, ZNG 6150; Distribution in Turkey: A1,A2, A4, B6, B7, C11, C13; VU (Finland, Ireland, Northern Ireland, Austria, Poland, Estonia), NT (Sicily). Ecological features: Subneutrophyte, hygrophyte, sciophyte; Cushion.

Pulvigera lyellii (Hook. \& Taylor) Plášek, Sawicki \& Ochyra - Loc: 5, 11, 12, 13, 15, 17, 23, 24, 26, 33, 44, 45; on tree trunk, ZNG 6150; Distribution in Turkey: A1, A2, A4, B6, B7, B8, C11, C12, C13; CR (Finland, Lithuania), EN (Belarus), VU (Estonia, Latvia), R (Poland), NT (Slovakia). Ecological features: Acidophyte, hygrophyte, photophyte; Tuft.

Ulota crispa (Hedw.) Brid - Loc: 15, 44, 51; on tree trunk, ZNG 6151; Distribution in Turkey: A1, A2, A3, A4, B6. Ecological features: Acidophyte, hygrophyte, photophyte; Cushion.

Zygodon rupestris Schimp. ex Lorentz - Loc: 29, 32; on tree trunk, ZNG 6152; Distribution in Turkey: A1, A2, B6, B9, C11, C12, C13; EN (Austria, Poland), VU (Switzerland, Hunhary, Lithuania), R (Latvia), NT (Czech Republic). Ecological features: Basiphyte, kserofit, sciophyte; Turf.

Aulacomniaceae Schimp.

Aulacomnium palustre (Hedw.) Schwägr. - Loc: 20; wetland on soil, ZNG 6153; Distribution in Turkey: A1, A2, B6, B7, B8; VU (Luxembourg), NT (Germany). Ecological features: Acidophyte, hygrophyte, photophyte; Tuft.

Climaciaceae Kindb.

Climacium dendroides (Hedw.) F. Weber \& D. Mohr - Loc: 10, 20, 39; on soil, ZNG 6154; Distribution in Turkey: A1, A2, A4, B6; VU (Netherlands). Ecological features: Acidophyte, hygrophyte, sciophyte; Dendroid.

Amblystegiaceae G. Roth.

Campyliadelphus chrysophyllus (Brid.) R.S. Chopra - Loc: 43; on soil covered rock, ZNG 6155; Distribution in Turkey: A1, A2, A4, B6, C11, C13; VU (Netherlands), NT (Germany). Ecological features: Subneutrophyte, mesophyte, sciophyte; Weft.

Campylium protensum (Brid.) Kindb. - Loc: 13, 28, 43; on rock and soil, ZNG 6190; Distribution in Turkey: A1, A2, A4; VU (Bulgaria), EN (Romania). Ecological features: Acidophyte, mesophyte, sciophyte; Mat rough.

Cratoneuron filicinum (Hedw.) Spruce - Loc: 5, 8, 48; on wet calcacreus soil and rock, ZNG 6156; Distribution in Turkey: A1, A2, A3, A4, A5, B6, B7, B8, B9, B10, C11, C12, C13, C15. Ecological features: Basiphyte, hygrophyte, photophyte; Weft.

Hygrohypnum luridum (Hedw.) Jenn. - Loc: 8, 16; stream bed on rocks, ZNG 6156; Distribution in Turkey: A1, A2, A4, B6; NT (Slovakia). Ecological features: Basiphyte, rheophyte, photophyte; Mat rough.

Palustriella commutata (Brid.) Ochyra - Loc: 5, 8, 48; on wet soil, ZNG 6157; Distribution in Turkey: A1, A2, A3, A4, B7, B9, B10, C11, C12, C13, C15; EN (Netherlands, Hungary), VU (Finland), NT (Sicily, Germany, Luxembourg). Ecological features: Basiphyte, hygrophyte, photophyte, Weft.

Pseudoamblystegium subtile (Hedw.) Vanderp. \& Hedenäs (Syn: Amblystegium subtile (Hedw.) Schimp., Platydictya subtilis (Hedw.) H.A.Crum) - Loc: 45; on tree trunk, ZNG 6158; Distribution in Turkey: A1, A2, A4; R (Poland), NT (Finland, Germany). Ecological features: Acidophyte, hygrophyte, sciophyte, Mat rough.

Serpoleskea confervoides (Brid.) Loeske (Syn: Amblystegiella confervoides (Brid.) Loeske, Amblystegium confervoides (Brid.) Schimp., Platydictya confervoides (Brid.) H.A. Crum) - Loc: 14, 28; on rock and tree trunk, ZNG 6159; Distribution in Turkey: A1, A2; NT (Ireland, Northern Ireland, Spain), VU (Hungary, Estonia). Ecological features: Acidophyte, hygrophyte, sciophyte; Mat rough.

Calliergonaceae Vanderpoorten, Hedenäs, C.J. ox \& A.J.Shaw. 
Calliergon cordifolium (Hedw.) Kindb - Loc: 20; on wet soil, ZNG 6160; Distribution in Turkey: A1, A2, A4, C13; EN (Austria), VU (Spain, Switzerland), NT (Hungary). Ecological features: Acidophyte, hygrophyte, sciophyte; Turf.

Sarmentypnum exannulatum (Schimper) Hedenas - Loc: 20; on wet soil, ZNG 6161; Distribution in Turkey: A1, A2, A4; CR (Luxembourg, Hungary), KW (Netherlands), VU (Slovenia). Ecological features: Acidophyte, rheophyte, photophyte; Turf.

Pseudoleskeaceae Schimp.

Lescuraea incurvata (Hedw.) E. Lawton - Loc: 14, 37, 38; on rock, ZNG 6162; Distribution in Turkey: A1, A2, A3, A4, B10, C11, C12, C13; CR (Hungary), VU (Great Britain), NT (Finland, Sicily, Germany). Ecological features: Acidophyte, kserofit, photophyte; Mat smooth.

Leskeaceae Schimp.

Pseudoleskeella catenulata (Brid. ex Schrad.) Kindb. - Loc: 9; on rock, ZNG 6163; Distribution in Turkey: A1, A2, A3, A4, C13; CR (Luxembourg), NT (Estonia). Ecological features: Basiphyte, kserofit, photophyte, Mat rough.

Pseudoleskeella nervosa (Brid.) Nyholm - Loc: 14, 23; on tree trunk, ZNG 6164; Distribution in Turkey: A1, A2, A4, B6, B8, C13; CR (Great Britain). Ecological features: Acidophyte, mesophyte, photophyte; Mat rough.

Thuidiaceae Schimp.

Abietinella abietina (Hedw.) M. Fleisch. - Loc: 8, 25, 26; on soil, ZNG 6165; Distribution in Turkey: A1, A2, A3, A4, B6, B9; EN (Ireland, Northern Ireland), V (Germany). Ecological features: Subneutrophyte, kserofit, photophyte; Weft.

Thuidium assimile (Mitt.) A. Jaeger - Loc: 11; on rock, ZNG 6166; Distribution in Turkey: A2, A3, A4. Ecological features: Subneutrophyte, hygrophyte, sciophyte; Weft

Brachytheciaceae G.Roth.

Brachytheciastrum velutinum (Hedw.) Ignatov \& Huttunen var. salicinum (Schimp.) Ochyra \& Zarnowiec Loc: 9, 14, 17, 48; on tree trunk ad soil, ZNG 6167; Distribution in Turkey: A1, A2, B6, C11, C12, C14. Ecological features: Acidophyte, mesophyte, sciophyte; Mat rough.

Brachytheciastrum velutinum (Hedw.) Ignatov \& Huttunen var. velutinum - Loc: 12; on soil, ZNG 6168; Distribution in Turkey: A1, A2, A3, A4, A5, B6, B7, B8, B10, C11, C12, C13, C14. Ecological features: Acidophyte, mesophyte, sciophyte; Mat rough.

Brachythecium albicans (Hedw.) Schimp. - Loc: 21; on soil, ZNG 6169; Distribution in Turkey: A1, A2, A3, A4, B6, B7, B8, C11, C13. Ecological features: Acidophyte, mesophyte, photophyte; Weft.

Brachythecium glareosum (Bruch ex Spruce) Schimp. - Loc: 13, 38; on soil, ZNG 6170; Distribution in Turkey: A1, A2, A3, A4, B6, B8, C11, C13; EN (Netherlands), NT (Hungary). Ecological features: Subneutrophyte, mesophyte, sciophyte; Mat rough.

Brachythecium mildeanum (Schimp.) Schimp. - Loc: 23; on soil covered rock, ZNG 6171; Distribution in Turkey: A1, A2, A3, A4, B8, B9, C13; EN (Austria), VU (Spain, Netherlands). Ecological features: Subneutrophyte, hygrophyte, photophyte; Weft.

Brachythecium rivulare Schimp. - Loc: 20, 24, 25; water resources on soil, ZNG 6172; Distribution in Turkey: A1, A2, A3, A4, A5, B6, B7, B8, B9, B10, C11, C12, C13; VU (Netherlands). Ecological features: Acidophyte, rheophyte, sciophyte, Mat rough.

Brachythecium rutabulum (Hedw.)Schimp. - Loc: 9, 16, 23; on rocks and tree trunks, ZNG 6173; Distribution in Turkey: A1, A2, A3, A4, B6, B8, C13. Ecological features: Acidophyte, hygrophyte, sciophyte; Weft.

Brachythecium salebrosum (F. Weber \& D. Mohr) Schimp. - Loc: 39, 48, 53; on rocks, soil and decaying log, ZNG 6174; Distribution in Turkey: A1, A2, A3, A4, B6, C11, C12, C13. Ecological features: Subneutrophyte, mesophyte, sciophyte; Mat rough.

Cirriphyllum crassinervium (Taylor) Loeske \& M. Fleisch. - Loc: 23; on soil covered rocks, ZNG 6175; Distribution in Turkey: A1, A2, A3, A4, B6, B7, C11, C12, C13. Ecological features: Subneutrophyte, hygrophyte, sciophyte; Mat rough.

Eurhynchiastrum pulchellum (Hedw.) Ignatov \& Huttunen (Syn: Eurhynchium pulchellum (Hedw.) Jenn.) Loc: 23; on soil, ZNG 6176; Distribution in Turkey: A1, A2, A3, A4, A5, B6, B8, B10, C11, C13; RE (Northern Ireland), EN (Great Britain, Austria), VU (Germany, Luxembourg), NT (Hungary). Ecological features: Acidophyte, mesophyte, sciophyte; Mat rough.

Eurhynchium angustirete (Broth.) T.J.Kop. - Loc: 39; on soil, ZNG 6177; Distribution in Turkey: A1, A2, A4, A5, C13; VU (Spain). Ecological features: Acidophyte, mesophyte, sciophyte; Weft.

Homalothecium lutescens (Hedw.) H. Rob. - Loc: 25, 29, 31; on rock and soil, ZNG 6178; Distribution in Turkey: A1, A2, A3, A4, B6, B7, B8, C11, C12, C13; NT (Finland). Ecological features: Subneutrophyte, mesophyte, photophyte; Weft.

Homalothecium philippeanum (Spruce) Schimp. - Loc: 8, 9, 14, 39; on rock, ZNG 6179; Distribution in Turkey: A1, A2, A3, A4, A5, B6, B7, B8, B9, B10, C11, C12, C13; NT (Germany). Ecological features: Basiphyte, kserofit, sciophyte; Mat rough. 
Homalothecium sericeum (Hedw.) Schimp. - Loc: 9, 11, 15, 16, 17, 23, 29, 39; on rock and tree trunk, ZNG 6180; Distribution in Turkey: A1, A2, A3, A4, A5, B6, B7, B8, B9, B10, C11, C12, C13, C14. Ecological features: Basiphyte, kserofit, sciophyte; Mat rough.

Kindbergia praelonga (Hedw.) Ochyra - Loc: 13, 30, 34, 36, 47; on soil, ZNG 6181; Distribution in Turkey: A1, A2, A3, A4, B6, B8, C11, C12, C13; VU (Finland, Slovakia, Hungary). Ecological features: Acidophyte, hygrophyte, sciophyte; Weft.

Microeurhynchium pumilum (Wilson) Ignatov \& Vanderp. (Syn: Eurhynchium pumilum (Wilson) Schimp., Oxyrrhynchium pumilum (Wilson) Loeske, Rhynchostegiella pumila (Wilson) E.F. Warb.) - Loc: 3, 11, 30, 32; on soil, ZNG 6182; Distribution in Turkey: A1, A2, A3, A4, B6, C11, C12, C13; RE (Austria), CR (Sweden, Romania), EN (Norway, Slovakia, Bulgaria), VU (Switzerland), NT (Canary Islands, Hungary). Ecological features: Subneutrophyte, kserofit, sciophyte; Mat rough.

Oxyrrhynchium hians (Hedw.) Loeske - Loc: 17, 29; on soil, ZNG 6183; Distribution in Turkey: A1, A2, A3, A4, B6, B7, B8, C11, C12, C13; NT (Canary Islands). Ecological features: Acidophyte, hygrophyte, sciophyte; Mat rough.

Oxyrrhynchium schleicheri (R. Hedw.) Roll - Loc: 28; on soil, ZNG 6184; Distribution in Turkey: A1, A2, A3, A4, B6, B8, C11, C13; CR (Ireland, Bulgaria), EN (Austria), VU (Sweden, Romania), NT (Hungary). Ecological features: Acidophyte, hygrophyte, sciophyte; Mat rough.

Pseudoscleropodium purum (Hedw.) M. Fleisch. - Loc: 40; on soil, ZNG 6185; Distribution in Turkey: A1, A2, A3, A4; NT (Finland, Canary Islands). Ecological features: Acidophyte, mesophyte, sciophyte; Weft.

Rhynchostegium riparioides (Hedw.) Cardot (Syn: Platyhypnidium riparioides (Hedw.) Dixon) - Loc: 1, 8, 9 , 51; stream bed on rock, ZNG 6186; Distribution in Turkey: A1, A2, A3, A4, A5, B6, B7, B8, B9, B10, C11, C12, C13; NT (Finland). Ecological features: Acidophyte, rheophyte, sciophyte; Mat rough.

Sciuro-hypnum populeum (Hedw.) Ignatov \& Huttunen (Syn: Brachythecium populeum (Hedw.) Bruch et al.) - Loc: 23, 35; on rock and soil, ZNG 6187; Distribution in Turkey: A1, A2, A3, A4, B6, B7, C11, C13. Ecological features: Acidophyte, mesophyte, sciophyte; Mat rough

Scleropodium touretii (Brid.) L.F. Koch - Loc: 41; on rocks and soil, ZNG 6188; Distribution in Turkey: A1, A2, A3, B6, B7, C11, C12; RE (Belgium), EN (Ireland, Northern Ireland, Bulgaria, Romania). Ecological features: Acidophyte, kserofit, sciophyte; Mat rough.

Hypnaceae Schimp.

Calliergonella cuspidata (Hedw.) Loeske - Loc: 8, 20, 25, 40; on wet soil, ZNG 6189; Distribution in Turkey: A1, A2, A3, A4, A5, B6, B7, B8, B9, C12, C13, C15; NT (Sicily). Ecological features: Acidophyte, hygrophyte, sciophyte; Weft.

Herzogiella seligeri (Brid.) Z.Iwats. - Loc: 9, 53; on decaying log and stump, ZNG 6191; Distribution in Turkey: A1, A2, A3, A4. Ecological features: Acidophyte, hygrophyte, sciophyte; Mat rough.

Hypnum andoi A.J.E. Sm. - Loc: 11, 12, 13, 15, 17, 24, 26, 27, 29, 35, 44, 52; on tree trunk, ZNG 6192; Distribution in Turkey: A1, A2, A3, A4, B6, C11, C13; VU (Serbia), NT (Sicily, Romania). Ecological features: Acidophyte, mesophyte, sciophyte; Mat smooth.

Hypnum cupressiforme Hedw. var. cupressiforme - Loc: 5, 11, 14, 18, 32, 33, 35, 39, 43, 44; on tree trunk, rocks and soil, ZNG 6193; Distribution in Turkey: A1, A2, A3, A4, A5, B6, B7, B8, C11, C12, C13. Ecological features: Acidophyte, mesophyte, sciophyte; Mat smooth.

Hypnum cupressiforme Hedw. var. lacunosum Brid. - Loc: 8, 11, 25, 33; on rock and soil, ZNG 6194; Distribution in Turkey: A1, A2, A3, A4, B6, B7, C11, C12, C13. Ecological features: Acidophyte, kserofit, sciophyte; Mat smooth.

Hypnum cupressiforme Hedw. var. resupinatum (Taylor) Schimp. - Loc: 17, 23; on tree trunk, ZNG 6195; Distribution in Turkey: A1, A2, A4, B6, B7, B8, C11, C13; NT (Sicily). Ecological features: Acidophyte, mesophyte, sciophyte; Mat rough.

Taxiphyllum wissgrillii (Garov.) Wijk \& Margad. - Loc: 17, 23; on soil covered rock, ZNG 6196; Distribution in Turkey: A1, A2, A4, B7; VU (Bulgaria). Ecological features: Basiphyte, hygrophyte, sciophyte; Mat smooth.

Pterigynandraceae Schimp.

Pterigynandrum filiforme Hedw. - Loc: 5, 9, 11, 12, 13, 14, 15, 17, 18, 21, 23, 24, 26, 27, 29, 32, 35, 39, 44, 45, 53; on rock and tree trunk, ZNG 6197; Distribution in Turkey: RE (Ireland, Northern Ireland, VU (Canary Isands, Luxembourg), NT (Germany). Ecological features: Subneutrophyte, mesophyte, sciophyte; Mat smooth.

Hylocomiaceae M. Fleisch.

Ctenidium molluscum (Hedw.) Mitt - Loc: 7, 11, 14, 16, 28, 30; on soil and rock, ZNG 6198; Distribution in Turkey: A1, A2, A3, A4, B6, C12, C13; EN (Netherlands), NT (Finland). Ecological features: Subneutrophyte, hygrophyte, sciophyte; Mat rough.

Pleurozium schreberi (Willd. ex Brid.) Mitt. - Loc: 20; on soil, ZNG 6199; Distribution in Turkey: A1, A2, A3, A4, A5. Ecological features: Acidophyte, mesophyte, sciophyte; Weft.

Rhytidiadelphus triquetrus (Hedw.) Warnst. - Loc: 10, 39; on soil, ZNG 6200; Distribution in Turkey: A1, A2, A3, A4, A5, B6; NT (Sicily). Ecological features: Subneutrophyte, hygrophyte, sciophyte; Weft. 
*Rhytidiadelphus subpinnatus (Lindb.) T.J.Kop. - Loc: 39; on soil, ZNG 6201; Distribution in Turkey: A4; NT (Great Britain), RE (Ireland), GE (Netherlands), VU (Romania). Ecological features: Subneutrophyte, hygrophyte, sciophyte; Weft.

Plagiotheciaceae (Broth.) M.Fleisch.

Plagiothecium cavifolium (Brid.) Z. Iwats. - Loc: 6, 45; on soil, ZNG 6202; Distribution in Turkey: A1, A2, A4; VU (Ireland). Ecological features: Acidophyte, hygrophyte, sciophyte; Mat smooth.

Plagiothecium curvifolium Schlieph. ex Limpr. - Loc: 22; on decaying log, ZNG 6203; Distribution in Turkey: A1, A2, A4, C13; VU (Ireland). Ecological features: Acidophyte, hygrophyte, sciophyte; Mat smooth.

Plagiothecium nemorale (Mitt.) A. Jaeger - Loc: 23, 46; on soil and decaying log, ZNG 6204; Distribution in Turkey: A1, A2, A3, A4, B8; NT (Finland). Ecological features: Acidophyte, hygrophyte, sciophyte; Mat smooth.

Plagiothecium succulentum (Wilson) Lindb. - Loc: 6, 7, 51; on soil and tree trunk, ZNG 6205; Distribution in Turkey: A1, A2, A3, A4; EN (Austria). Ecological features: Acidophyte, mesophyte, sciophyte; Mat smooth.

Leucodontaceae Schimp.

Antitrichia curtipendula (Timm ex Hedw.) Brid. - Loc: 9, 15; on tree trunk, ZNG 6206; Distribution in Turkey: A1, A2, A3, A4, B6, B7, B8, C11; RE (Poland), EN (Austria, Netherlands, Hungary, Lithuania), VU (Germany, Slovakia, Latvia), NT (Ireland, Northern Ireland, Estonia). Ecological features: Acidophyte, hygrophyte, sciophyte; Weft.

Leucodon sciuroides (Hedw.) Schwägr. Loc: 10, 11, 12, 14, 15, 16, 17, 21, 23, 26, 33, 35, 39, 44, 45, 53; on tree trunk and rock, ZNG 6207; Distribution in Turkey: A1, A2, A3, A4, A5, B6, B7, B8, C11, C12, C13. Ecological features: Acidophyte, mesophyte, photophyte; Mat rough.

Neckeraceae Schimp.

Alleniella besseri (Lobarz.) S.Olsson, Enroth \& D.Quandt - Loc: 16, 23; on tree trunk, ZNG 6208; Distribution in Turkey: A1, A2, A4, C12; EN (Switzerland), VU (Germany), R (Poland), NT (Finland, Sweden). Ecological features: Subneutrophyte, kserofit, sciophyte; Fan.

Alleniella complanata (Hedw.) S. Olsson, Enroth \& D.Quandt (Syn: Neckera complanata (Hedw.) Huebener) - Loc: 9, 11, 14, 16, 39; on tree trunk and rock, ZNG 6209; Distribution in Turkey: A1, A2, A3, A4, B6, C13; EN (Netherlands), VU (Latvia). Ecological features: Acidophyte, mesophyte, sciophyte; Fan.

Exsertotheca crispa (Hedw.) S. Olsson, Enroth \& D. Quandt (Syn: Neckera crispa Hedw.) - Loc: 14; on rock, ZNG 6210; Distribution in Turkey: A1, A2, A3, C13; EN (Netherlands), R (Latvia, Lithuania), NT (Sicily, Hungary). Ecological features: Subneutrophyte, mesophyte, sciophyte; Fan.

Neckera menziesii Drumm. (Syn: Metaneckera menziesii (Drumm.) Steere) - Loc: 9; on rock, ZNG 6211; Distribution in Turkey: A1, A2, B6, B8, C11, C12, C13; CR (Czech Republic, Luxembourg, Slovakia, Switzerland, Romania), EN (Austria, Germany), VU (Canary Islands). Ecological features: Subneutrophyte, mesophyte, sciophyte; Fan.

Neckera pumila Hedw. - Loc: 14; on rock, ZNG 6212; Distribution in Turkey: A1, A2, B7; RE (Czech Republic), EN (Finland, Austria, Germany, Netherlands, Poland, Slovakia, Bulgaria), VU (Switzerland, Romania), NT (Sweden, Sicily). Ecological features: Acidophyte, mesophyte, sciophyte; Mat smooth.

Thamnobryum alopecurum (Hedw.) Gangulee - Loc: 1, 9, 14, 15, 51; on rock, ZNG 6213; Distribution in Turkey: A1, A2, A3, A4, B7, C11, C12; EN (Latvia), VU (Canary Islands), NT (Estonia). Ecological features: Subneutrophyte, hygrophyte, sciophyte; Dendroid.

Lembophyllaceae Broth.

Isothecium alopecuroides (Lam. ex Dubois) Isov. - Loc: 6, 14, 15, 16, 23, 24, 26, 44, 45, 5; on tree trunk, ZNG 6214; Distribution in Turkey: A1, A2, A3, A4, B6, B7, C11, C13; VU (Netherlands). Ecological features: Acidophyte, mesophyte, sciophyte; Dendroid.

Isothecium myosuroides Brid. - Loc: 44; on tree trunk, ZNG 6215; Distribution in Turkey: A1, A2, A3, A4, C13; CR (Bulgaria), EN (Hungary, Latvia), NT (Switzerland, Estonia). Ecological features: Acidophyte, hygrophyte, sciophyte; Dendroid

\section{Anomodontaceae Kindb.}

Anomodon attenuatus (Hedw.) Huebener. - Loc: 11, 14, 16, 29, 43, 49; on rock and tree trunk, ZNG 6215; Distribution in Turkey: A1, A2, A3, A4, B6; EN (Great Britain, Netherlands). Ecological features: Subneutrophyte, mesophyte, sciophyte; Mat rough.

Anomodon viticulosus (Hedw.) Hook. \& Taylor - Loc: 9, 14, 16, 23, 49; on rock and tree trunk, ZNG 6216; Distribution in Turkey: A1, A2, A3, A4, B6, C13; EN (Netherlands), NT (Sicily). Ecological features: Subneutrophyte, mesophyte, sciophyte; Mat rough.

\section{Conclusions and discussion}

According to this bryofloristic list, 11 taxa (Anthoceros caucasicus Steph., Isopaches bicrenatus (Schmidel ex Hoffm.) H.Buch, Marchantia polymorpha subsp. montivagans Bischl. \& Boisselier, Riccia sorocarpa Bisch., Sphagnum contortum Schultz, Entosthodon pulchellus (H. Philib.) Brugués, Grimmia nutans Bruch, Schistidium papillosum Culm., Schistidium robustum (Nees \& Hornsch.) H.H. Blom, Ephemerum crassinervium subsp. sessile (Bruch) Holyoak and 
Rhytidiadelphus subpinnatus (Lindb.) T.J.Kop.) are new records for the A2 grid-square according to the Henderson's Turkish grid square system [8]. Moreover, S. contortum Schultz is secondly reported in Turkey with this study. Hitherto, it was known only from Anzer plateau of Rize province in Turkey [40]. Nevertheless, this taxon was collected abundantly in wetland areas at the Turnalı Plateau $(1410 \mathrm{~m})$ on 21 May 2017 in Akyazı district of Sakarya province. So that, this record contributes a range extension of approximately $1000 \mathrm{~km}$ to the north-west of Turkey from the first recorded location. Moreover, it has been classified as vulnerable (VU) for Spain, Slovakia, Hungary, Montenegro, Serbia in the Red data book of European bryophytes. This species is different from other the members of Sect. Subsecunda (except S. platyphyllum) with its 2-3 layered stem cortex and pale stem. In addition, the most similar species; S. platyphyllum has bigger stem and branch leaves than $S$. contortum, and also, the number of branches on fassicles in $S$. contortum are more than $S$. platyphyllum [40, 41]. Accompanying moss species to $S$. contortum in this locality were hygrophytic species such as; Chiloscyphus polyanthos (L.) Corda, Polytrichum commune Hedw., Imbribryum alpinum (Huds. ex With.) N. Pedersen, Philonotis caespitosa Jur., Philonotis capillaris Lindb., Aulacomnium palustre (Hedw.) Schwägr. and Calliergon cordifolium (Hedw.) Kindb.

Furthermore, in general, in terms of the number of taxon rich families in the research area are respectively; Brachytheciaceae (22 taxa), Pottiaceae (18 taxa), Orthotrichaceae (12 taxa), Mniaceae (11 taxa) for mosses, and Lophocoleaceae (4 taxa) and Plagiochilaceae (2 taxa), Scapaniaceae (2 taxa), Lejeuneaceae (2 taxa), Radulaceae (2 taxa), Aneuraceae (2 taxa), Metzgeriaceae (2 taxa), Marchantiaceae (2 taxa) for liverworts. However hornworts are represented by only one family and genus.

If we compare taxa in the area with their ecological characteristics; hygrophyte mosses (46\%) were dominantly found at especially western and northern parts of the research area. After that, mesophytic (35\%), xerophytic (16\%) and others $(3 \%)$ taxa were generally seen at the eastern, southern parts and summits of the region. According to the light preferences of the bryophytes in this region, the majority rate of them belong to sciophytes $(68 \%)$. The remainder of taxa are photophytes (32\%). While acidophilic conditions take the first place in the substrate preferences of bryophytes in this region $(58 \%)$, the other preferences are namely; subneutrophytic (32\%) and basophilic (10\%) substrates. According to our efforts to determine the ecological habitats of bryophytes terricolous bryophyte species were found very common $(47 \%)$ in the research area and others are respectively; saxicolous (20\%), corticolous (14\%), lignicolous (4\%), and facultative $(16 \%)$. Considering the percentages of life forms of bryophytes prevailing in the field, the ranking are as follows; Turf (33\%), Mat Smooth (15\%), Mat rough (15\%), Weft (10\%), Cushion (8\%), Tuft (7\%), Mat Thalloid (6\%) and the other life forms $(6 \%)$. Finally, the authors compared with checklist and country status of European Bryophytes Red List in the floristic list of Bryophytes [42]. As a result of this comparison, we have determined that; in our floristic list 22 taxa in regionally extinct (RE), 24 taxa in critically endangered (CR), 66 taxa in endangered (EN), 103 taxa in vulnerable (VU), 6 taxa in rare (R) and 109 taxa in near threatened (NT) were listed by different European countries. This situation shows us that the study area has a very valuable bryofloristic richness. In our opinion, limitation of intensive anthropogenic activities in this region is important for the preservation of the rich bryophyte diversity. As you known, they colonize the barren rocks and exposed areas of hills, and make them suitable for growing angiosperm and other plants by depositing humus soil and dead plant scraps. After that, the forms and grasses grow, and after all shrubs and trees also settle down, and the whole area changes into dense wood. Furthermore a few bryophytes play an important role by their extensive carpets, and prevent the soil erosion to some extent. Moreover, bryophytes have been used in medicine for along time especially in Far East countries, because of their extracts contain phenolic compounds that inhibit growth of pathogenic fungi and bacteria [43-47].

\section{References}

[1] Konrat, M., Shaw, A.J., Renzaglia, K.S. (2010). A special issue of Phytotaxa dedicated to Bryophytes: The closest living relatives of early land plants. Phytotaxa, 9: 5-10. http://dx.doi.org/10.11646/phytotaxa.9.1

[2] Ezer, T., Zander, R.H. (2017). Tortula galilaea (Herrnst. Heyn) T. Ezer R.H.Zander comb. nov. (Pottiaceae, Bryophyta). Journal of Bryology, 39 (2): 207-209. https://doi.org/10.1080/03736687.2016.1209275.

[3] Yücel, E., Ezer, T. (2017). New national and regional bryophyte records, 53, 14. Entosthodon hungaricus (Boros) Loeske. Journal of Bryology, 39 (4): 6-7. https://doi.10.1080/03736687.2017.1384204.

[4] Uyar, G., Ören, M., Ezer, T., Can Gözcü, M. (2018). The genus Pseudephemerum and Schistidium confusum newly reported from Turkey and Southwestern Asia. Cryptogamie Bryologie, 39 (1): 55-60. https://doi.org/10.7872/cryb/v39.iss1.2018.55

[5] Alataş, M. \& Uyar, G. (2015). The Bryophyte Flora of Abant Mountains (Bolu/Turkey). Biological Diversity and Conservation, 8 (1): 35-43.

[6] Gürsu, G. \& Çetin, B. (2017). Karasu (Sakarya/Türkiye) ve Civarının Karayosunu (Briyofit) Florası. Anatolian Bryology, 3 (2): 68-74. https://doi. 10.26672/anatolianbryology.347796.

[7] Can Gözcü, M., Uyar, G., Ören, M., Ezer, T. \& Alataş, M. (2019). The Bryophyte Flora of the Samanlı Mountains (Sakarya, Kocaeli, Yalova, Bursa) in North-West Turkey. Arctoa, 28: 58-74. https://doi.10.5281/zenodo.1468239. 
[8] Henderson, D.M. (1961). Contribution to the Bryophyte Flora of Turkey: IV. Notes from Royal Botanic Garden Edinburgh, 23: 263-278.

[9] Akman, Y. (2011) İklim ve Biyoiklim (Biyoiklim metodlarl ve Türkiye İklimleri). Ankara, TR: Palme Yayıncılık.

[10] Tsms (Turkish State Meteorological Service), (2016). Sakarya weather station climate data (1950-2015). Ankara, TR: Research and Computing Department.

[11] Zohary, M. (1973). Geobotanical Foundations of the Middle East Band 1-2. Amsterdam, DE: Gustave Fischer Verlag.

[12] Karbuz, İ. (2015). Sakarya Nehri ve Doğançay Deresi Arasindaki Sahada Bitki Örtüsünün Ekolojik Şartları. Akademik Sosyal Araştırmalar Dergisi, 3 (19): 345-364. https://doi. 10.16992/ASOS.933

[13] Smith, A.J.E. (2004). The Moss Flora of Britain and Ireland. Cambridge, GB: Cambridge Univ. Press.

[14] Paton, J. (1999). The Liverworts Flora of the British Isles. Oxon, GB: Harley Books.

[15] Cortini Pedrotti, C. (2001). Flora dei muschi d'Italia, Sphagnopsida, Andreaopsida, Bryopsida (I parte). Roma, IT: Antonio Delfino Editore Medicina-Scienze.

[16] Cortini Pedrotti, C. (2006). Flora dei muschi d'Italia, Bryopsida (II parte). Roma, IT: Antonio Delfino Editore Medicina-Scienze.

[17] Greven, H.C. (2003). Grimmias of the World. Leiden, NL: Backhuys Publishers.

[18] Heyn, C.C. \& Herrnstadt, I. (2004). The Bryophyte Flora of Israel and Adjacent Regions. Jerusalem, IL: The Israel Academy of Sciences and Humanities.

[19] Frey, W., Frahm, J.P., Fischer, E., Lobin, W. (2006). The liverworts, mosses and ferns of Europe. Essex, GB: Harley Books.

[20] Guerra, J., Cano, M.J., Cros, R.M. (2006). Flora Briofitica Iberica VoIumen 3, Pottiales: Pottiaceae, Encalyptales: Encalyptaceae. Murcia, ES: Uniersidad de Murcia, Sociedad Espanola de Briyologia.

[21] Guerra, J. \& Cros, M. (2007). Flora Briofitica Ibérica. Vol. 1. Murcia, ES: Universidad de Murcia Sociedad Española de Briología.

[22] Casas, C., Brugués, M., Cros, M.R., Sérgio, C., Infante, M. (2009). Handbook of Liverworts and Hornworts of the Iberian Peninsula and the Balearic Islands. Barcelona, ES: Institut d'Estudis Catalans.

[23] Kürschner, H. \& Frey, W. (2011). Liverworts, Mosses and Hornworts of Southwest Asia. Stuttgart, DE: Beiheft 139.

[24] Plášek, V., Sawicki, J., Ochyra, R., Szczecińska, M., Kulik, T. (2015). New taxonomical arrangement of the traditionally conceived genera Orthotrichum and Ulota (Orthotrichaceae, Bryophyta). Acta Mus. Siles. Sci. Natur., 64: 169-174. https://doi.10.1515/cszma-2015-0024

[25] Lara, F., Garilleti, R., Goffinet, B., Draper, I., Medina, R., Vigalondo, B., Mazimpaka, V. (2016). Lewinskya, a new genus to accommodate the phaneroporous and monoicous taxa of Orthotrichum (Bryophyta, Orthotrichaceae). Cryptogamie Bryologie, 37: 361-382. https://doi.org/10.7872/cryb/v37.iss4.2016.361.

[26] Uyar, G. \& Çetin, B. (2004). A new check-list of the mosses of Turkey. Journal of Bryology, 26: $203-220$.

[27] Kürschner, H. \& Erdağ, A. (2005). Bryophytes of Turkey: An annotated reference list of the species with synonyms from the recent literature and an annotated list of Turkish bryological literature. Turkish Journal of Botany, 29: 95-154.

[28] Ros, R.M., Mazimpaka, V., Abou-Salama, U., Aleffi, M., Blockeel, T.L. et al (2007). Hepatics and Anthocerotes of the Mediterranean, an annotated checklist. Cryptogamie Bryologie, 28 (4): 351-437.

[29] Ros, R.M., Mazimpaka, V., Abou-Salama, U., Aleffi, M., Blockeel, T.L. et al (2013). Mosses of the Mediterranean, an annotated checklist. Cryptogamie Bryologie, 34 (2): 99-283. https://doi: 10.782/cryb.v34.iss2.2013.99.

[30] Özenoğlu Kiremit, H., Keçeli, T. (2009). An annotated check-list of the Hepaticae and Anthocerotae of Turkey. Cryptogamie Bryologie, 30 (3): 343-356.

[31] Erdağ, A. \& Kürschner, H. (2017). Türkiye Bitkileri Listesi (Karayosunları). İstanbul, TR: Ali Nihat Gökyiğit Vakfi Yayınları.

[32] Goffinet, B., Shaw, A.J. (2009). Bryophyte Biology, Second Edition. Cambridge, UK: Cambridge University Press.

[33] Crandall-Stotler, B., Stotler, R.E. \& Long, D.G. (2009). Phylogeny and classification of the Marchantiophyta. Edinburgh Journal of Botany, 66: 155-198. https://doi: 10.1017/S0960428609005393

[34]. Renzaglia, K.S. Villarreal, J.C. \& Duff, R.J. (2008). New insights into morphology, anatomy, and systematics of hornworts. In Bryophyte Biology. 2nd ed. Goffinet, B. Shaw, AJ (eds.). Cambridge, NY: Cambridge University Press. 
[35] Ursavaş, S. \& Abay, G. (2009). The mosses (Musci) check-list for A2 square of Turkey. Bartın Orman Fakültesi Dergisi, 11 (16): 33-43.

[36] Ören, M., Uyar, G., Keçeli, T. (2012). The bryophyte flora of the western part of the Küre Mountains (Bartın, Kastamonu), Turkey. Turkish Journal of Botany, 36: https://doi: 538-557. 10.3906/bot-1111-2.

[37] Mägdefrau, K. (1982). Life forms of bryophytes. In: Smith AJE (ed.) Bryophyte Ecology, London, UK: Chapman and Hall.

[38] Hill, M.O., Preston, C.D., Bosanquet, S.D.S., Roy, D.B. (2007). Bryoatt, attributes of British and Irish mosses, liverworts and hornworts with information on native status, size, life form, life history, geography and habitat. Norwich: UK, NERC Copyright,

[39] Dierßen, K. (2001). Distribution, ecological amplitude and phytosociological characterization of European bryophytes. Stuttgart, DE: Bryophytorum Bibliotheca.

[40] Kirmac1, M., Kürschner, H. (2013). The genus Sphagnum L. in Turkey - with S. contortum, S. fallax, S. magellanicum and S. rubellum, new to Turkey and Southwest Asia. Nova Hedwigia, 96 (3-4): 383-397. https://doi: 10.1127/0029-5035/2013/0079.

[41] Laine, J., Harju, P., Timonen, T., Laine, A., Tuittila, E.S., Minkkinen, K., Vasander, H. (2011). The Intricate Beauty of Sphagnum Mosses: A Finnish Guide to Identification. Second edition, Department of Forest Sciences, University of Helsinki, Finland.

[42] Hodgetts, N.G. (2015). Checklist and country status of European bryophytes- towards a new Red List for Europe. Ireland: Irish Wildlife Manuals, No. 84. National Parks and Wildlife Service, Department of Arts, Heritage and the Gaeltacht.

[43] Dülger, B., Hacioglu, N. \& Uyar, G. (2009). Evaluation of Antimicrobial Activity of Some Mosses from Turkey. Asian Journal of Chemistry, 21 (5): 4093-4096.

[44] Uyar, G., Hacioğlu Doğru, N., Ören, M. \& Çavuş, A. (2016). Determining Antibacterial Activity of Some Mosses (Cinclidotus riparius (Host ex Brid.) Arn., Calliergonella cuspidata (Hedw.) Loeske, Thamnobryum alopecurum (Hedw.) Gangulee, Leucobryum juniperoideum (Brid.) Müll.Hal., Cirriphyllum crassinervium (Taylor) Loeske \& M.Fleisch.). Anatolian Bryology, 1-2 (2): 1-8.

[45] Alan, Ş., Koçer, F. \& Uyar, G. (2018). Antimicrobial Activities of Some Bryophytes in Turkey. Commun. Fac. Sci. Univ. Ank. Series C, 27 (2): 17-22.

[46] Hazer, Y., Çölgeçen, H. \& Uyar, G. (2017). Briyofitlerden Elde Edilen Fenolik Bileşikler. Karaelmas Fen ve Müh. Derg., 7(1): 333-340.

[47] Hazer, Y., Çölgeçen, H., Koca-Çalışkan, U. \& Uyar, G. (2019). Thamnobryum alopecurum'un in vitro Doku Kültürü, Fitokimyasal İçeriği, Biyolojik Aktivitesi. Karaelmas Fen ve Müh. Derg., 9 (1): 1-14 https://doi: 10.7212/zkufbd.v9i1.1480. 